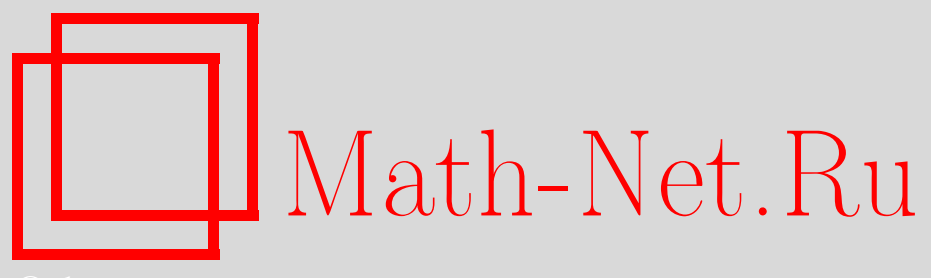

А. А. Гончар, Е. А. Рахманов, С. П. Суетин, Аппроксимации Паде-Чебышёва для многозначных аналитических функций, вариация равновесной энергии и $S$ свойство стационарных компактов, УМH, 2011, том 66, выпуск 6, 3-36

DOI: https://doi.org/10.4213/rm9452

Использование Общероссийского математического портала Math-Net.Ru подразумевает, что вы прочитали и согласны с пользовательским соглашением http://www . mathnet.ru/rus/agreement

Параметры загрузки:

IP: 54.198 .67 .100

26 апреля 2023 г., 05:55:07

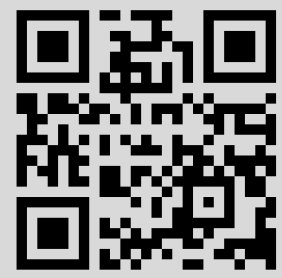




\title{
Аппроксимации Паде-Чебышёва для многозначных аналитических функций, вариация равновесной энергии и $S$-свойство стационарных компактов
}

\author{
А. А. Гончар, Е. А. Рахманов, С. П. Суетин
}

Рассматриваются аппроксимации Паде-Чебышёва для многозначных аналитических функций, заданных и вещественных на единичном отрезке $[-1,1]$. Основное внимание уделяется нелинейным аппроксимациям Паде-Чебышёва. Для таких рациональных аппроксимаций получен аналог классической теоремы Шталя о сходимости по емкости аппроксимаций Паде в соответствующей "максимальной” области голоморфности заданной функции. Скорость сходимости характеризуется в терминах стационарного компакта для смешанной (гриново-логарифмической) теоретико-потенциальной задачи равновесия.

Библиография: 79 названий.

Ключевые слова: рациональные аппроксимации, аппроксимации Паде, многочлены Чебышёва, нелинейные аппроксимации Паде-Чебышёва, стационарный компакт, теорема Шталя, сходимость по емкости.

\section{СоДЕРЖАНИЕ}

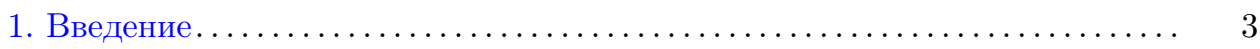

2. Обозначения и формулировка основных результатов ............. 13

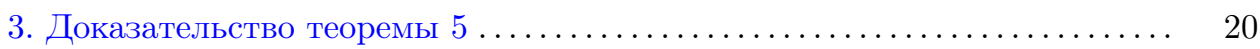

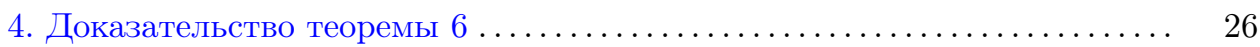

Список литературы ................................... 31

\section{1. Введение}

1.1. Пусть на единичном отрезке $E=[-1,1]$ задана вещественная функция $f$, голоморфная на $E$. Для произвольного натурального числа $n$ через $\mathscr{R}_{n}$ обозначим класс всех рациональных функций $r$ вида $r=p / q$, где $p, q$ - произвольные алгебраические полиномы степени $\leqslant n, q \not \equiv 0$. Отметим, что число свободных параметров, определяющих функцию из класса $\mathscr{R}_{n}$, равно $2 n+1$.

Работа выполнена при поддержке РФФИ (грант № 11-01-00330) и программы "Ведущие научные школы" (грант НШ-8033.2010.1).

(C) А. А. Гончар, Е. А. РАхманов, С. П. Суетин, 2011 
Пусть функция $f$ продолжается с отрезка $E$ в некоторую область $G \supset E$ как (однозначная) голоморфная функция. Тогда для величины

$$
\varepsilon_{n}(f)=\inf _{r \in \mathscr{R}_{n}}\|f-r\|_{E}=\inf _{r \in \mathscr{R}_{n}} \sup _{x \in E}|(f-r)(x)|
$$

- наилучшего равномерного приближения $f$ рациональными дробями из класса $\mathscr{R}_{n}$ - справедливо неравенство Уолша (см. [1]):

$$
\varlimsup_{n \rightarrow \infty} \varepsilon_{n}^{1 / n} \leqslant q,
$$

где

$$
q=\exp \left\{-\frac{1}{C(E, \overline{\mathbb{C}} \backslash G)}\right\}<1,
$$

$C(E, \overline{\mathbb{C}} \backslash G)$ - емкость конденсатора $(E, \overline{\mathbb{C}} \backslash G)$.

Неравенство (2) носит универсальный характер - оно неулучшаемо в классе всех функций, голоморфных в области $G$. Однако для некоторых вполне естественных классов функций неравенство Уолша (2) может быть существенно усилено.

Пусть $f=\widehat{\sigma}$, где $\widehat{\sigma}-$ марковская функиия:

$$
\widehat{\sigma}(z)=\int_{c}^{d} \frac{d \sigma(x)}{z-x}, \quad z \in \overline{\mathbb{C}} \backslash[c, d],
$$

$\sigma$ - положительная борелевская мера на отрезке $[c, d] \subset \mathbb{R},[c, d] \cap E=\varnothing$. Если $\sigma^{\prime}=d \sigma / d x>0$ почти всюду (п. в.) на $[c, d]$, то для наилучших рациональных аппроксимаций функции $f$ имеем (см. [2])

$$
\lim _{n \rightarrow \infty} \varepsilon_{n}^{1 / n}=q^{2}
$$

где величина $q<1$ имеет тот же смысл, что и в $(2)$, с заменой конденсатора $(E, \overline{\mathbb{C}} \backslash G)$ на конденсатор $(E,[c, d])$ (в соотношении (5) содержатся два утверждения: существование предела и информация о его величине). Таким образом, в классе марковских функций (4), голоморфных в области $G=\overline{\mathbb{C}} \backslash[c, d]$, вместо неравенства Уолша (2) имеет место существенно более сильное равенcтво (5). Соотношение (5) справедливо и для функций вида $f=\widehat{\sigma}+r$, где $r-$ рациональная функций, голоморфная на $E$.

В серии работ 1985-1986 гг. Г. Шталь [3]-[7] (см. также [8], [9]) получил ряд результатов о сходимости классических и многоточечных аппроксимаций Паде для функций, голоморфных соответственно в точке $z=\infty$ и на фиксированном односвязном континууме в $\mathbb{C}$ и имеющих в $\overline{\mathbb{C}}$ конечное число особых точек многозначного характера. Из его результата, относящегося к случаю континуума, и результатов работы $\left[10 ; \S 1\right.$, теорема $1^{\prime}$, формула $\left.(28)\right]$ вытекает справедливость равенства (5) в классе функций, вещественных и голоморфных на $E=[-1,1]$ и имеющих в $\overline{\mathbb{C}} \backslash E$ конечное число особых точек многозначного характера (см. ниже определение 1 ). Точнее, обозначим через $R_{n}$ наилучшие равномерные рациональные аппроксимации функции $f$ на отрезке $E$ в классе $\mathscr{R}_{n}$. Тогда из “второй" теоремы Шталя (см. [6]-[7], а также [11; гл. 1, §3]) и теоремы $1^{\prime}$ работы $[10 ; \S 1$, формула (28)] вытекает справедливость следующего результата. 
Теорема 1. Пусть функиия $f$ вещественна и голоморфна на отрезке $E=$ $[-1,1]$ и допускает голоморфное продолжение с отрезка в $\overline{\mathbb{C}}$ всюду за исключением конечного числа особых точек многозначного характера. Тогда существует единственный компакт $F=F(f)$, не пересекающийся с $E$ и такой, что функиия $f$ голоморфна ${ }^{1}$ в области $\overline{\mathbb{C}} \backslash F$ и для любого компакта $K \subset \overline{\mathbb{C}} \backslash(E \cup F)$

$$
\left|\left(f-R_{n}\right)(z)\right|^{1 / n} \stackrel{\text { cap }}{\longrightarrow} e^{-2 G_{F}^{\lambda}(z)}<1, \quad z \in K .
$$

(Здесь " $\stackrel{\text { сар }}{\longrightarrow}$ ” означает сходимость по логарифмической емкости на соответствующем компакте.) В соотношении (6) $\lambda=\lambda^{F}$ - единичная мера с носителем на $E, G_{F}^{\lambda}(z)$ - гринов (относительно $F$ ) потенциал меры $\lambda$. Компакт $F$ обладает так называемым $S$-свойством (см. $(21))$, не разбивает плоскость и состоит из конечного числа аналитических дуг, а $\lambda$ - равновесная мера (с носителем на $E$; см. раздел 2). Соотношение (6) означает, что последовательность $R_{n}$ сходится к функции $f$ в области Шталя $D_{f}:=\overline{\mathbb{C}} \backslash F$ со скоростью геометрической прогрессии. С учетом результатов работы [10] из (6) вытекает, что в условиях и обозначениях теоремы 1 справедлив аналог равенства (5)

$$
\lim _{n \rightarrow \infty} \varepsilon_{n}^{1 / n}=q^{2}
$$

величина $q$ имеет тот же смысл, что и в $(2)$, с заменой конденсатора $(E, \overline{\mathbb{C}} \backslash G)$ на конденсатор $(E, F), F=F(f)$. Отметим, что $S$-симметричный компакт $F$ является стационарной точкой некоторого функционала энергии (см. раздел 2).

Обозначим через $\mu(Q)$ меру, ассоциированную с произвольным полиномом $Q$ :

$$
\mu(Q)=\sum_{\zeta: Q(\zeta)=0} \delta_{\zeta},
$$

где $\delta_{\zeta}$ - мера Дирака с носителем в точке $\zeta$. Пусть $\widetilde{\lambda}=\beta_{F}(\lambda)$ - выметание равновесной меры $\lambda$ из области Шталя $D_{f}=\overline{\mathbb{C}} \backslash F$ на $F=\partial D_{f}$. Тогда в условиях теоремы Шталя для знаменателей $q_{n}(z)$ рациональных функций $R_{n}$ имеем:

$$
\frac{1}{n} \mu\left(q_{n}\right) \rightarrow \tilde{\lambda}, \quad n \rightarrow \infty
$$

где сходимость мер понимается в слабой топологии. Таким образом, мера $\tilde{\lambda}$ (с носителем на $F$ ) характеризует предельное распределение полюсов рациональной аппроксимации $R_{n}$, а сама равновесная мера $\lambda$ (с носителем на $E$ ) характеризует предельное распределение точек интерполяции функции $f$ рациональной функцией $R_{n}$ (cp. (40)).

В связи с (8) напомним хорошо известную теорему Йенча-Сегё (см. [12], [13], а также [14]-[16]) для наилучших равномерных полиномиальных аппроксимаций функции $f$, голоморфной на $E$.

\footnotetext{
${ }^{1}$ Точнее, функция $f$ допускает голоморфное продолжение в $\overline{\mathbb{C}} \backslash F$, которое мы также будем обозначать через $f$.
} 
Для произвольного $\rho>1$ через $\gamma_{\rho}$ обозначим эллипс с фокусами в точках \pm 1 и суммой полуосей, равной $\rho$. Внутренность эллипса $\gamma_{\rho}$ обозначим через $G_{\rho}$; области $G_{\rho}$ будем называть каноническими (относительно $E$ ). Пусть $\rho_{0}=$ $\rho_{0}(f)$ - индекс максимальной канонической области, в которую $f$ продолжается как голоморфная функция.

ТЕОРЕМА ЙЕНЧА-СЕГЁ. Пусть функиия $f$ голоморфна на отрезке Е. Тогда существует бесконечная подпоследовательность $\Lambda=\Lambda(f) \subset \mathbb{N}$ такая, что для наилучших равномерных полиномиальных аппроксимачий $P_{n}$ функиии $f$ имеет место соотношение:

$$
\frac{1}{n} \mu\left(P_{n}\right) \rightarrow \lambda_{\gamma}, \quad n \in \Lambda, \quad n \rightarrow \infty
$$

где

$$
d \lambda_{\gamma}(\zeta)=\frac{1}{2 \pi} \frac{|d \zeta|}{\left|\zeta^{2}-1\right|^{1 / 2}}, \quad \zeta \in \gamma
$$

- равновесная мера для эллипса $\gamma=\gamma_{\rho_{0}(f)}, \rho_{0}(f)$ - индекс голоморфности функuиu $f$.

Таким образом, нули полиномов наилучшего равномерного приближения функции $f$ локализуют максимальный эллипс голоморфности $f$, фактически "отрезая" внутренность этого эллипса от его внешней части. Аналогичный результат справедлив и для наилучших равномерных рациональных аппроксимаций функции $f$ с фиксированной степенью знаменателя; подробнее см., например, [16].

Отметим, что хорошо известный алгоритм Ремеза [17] (см. также [18]-[24]) позволяет практически находить наилучшие равномерные полиномиальные и рациональные аппроксимации заданной функции. Именно этот алгоритм реализован в системе Maple (см. [25]). Однако процесс практического приближенного построения такой рациональной аппроксимации предполагает, что исходная функция $f$ задана в виде явного аналитического (формульного) выражения.

1.2. Пусть $T_{k}(x)$ - полиномы Чебышёва (первого рода), ортонормированные на отрезке $E$ по мере $d \tau(x)=\left(1-x^{2}\right)^{-1 / 2} d x$. Функция $f, f \in \mathscr{H}(E)$, представляется на $E$ рядом Фурье-Чебышёва:

$$
f(x)=\sum_{k=0}^{\infty} c_{k} T_{k}(x), \quad c_{k}=c_{k}(f)=\int_{-1}^{1} f(x) T_{k}(x) d \tau(x) .
$$

Ряд (9) сходится к $f(x)$ локально равномерно в области $G_{0}(f)=G_{\rho}$, где $\rho=$ $\rho_{0}(f)$ - индекс максимальной канонической области, в которую $f$ продолжается как голоморфная функция. Область $G_{0}(f)$ - максимальная область сходимости ряда (9).

Будем теперь считать, что вещественная функция $f \in \mathscr{H}(E)$ задана на $E$ (сходящимся) рядом Фурье-Чебышёва

$$
f(x)=\sum_{k=0}^{\infty} c_{k} T_{k}(x), \quad c_{k}=c_{k}(f) \in \mathbb{R}
$$


(см. [26]-[29] и ниже замечание 1 ). Для частичных сумм $S_{n}$ ряда (10) также справедлив аналог теоремы Йенча-Сегё:

$$
\frac{1}{n} \mu\left(S_{n}\right) \rightarrow \lambda_{\gamma}, \quad n \in \Lambda^{\prime}=\Lambda^{\prime}(f), \quad n \rightarrow \infty .
$$

Таким образом, нули частичных сумм ряда (10) в пределе также локализуют максимальный эллипс голоморфности $f$ (см. рис. 1).

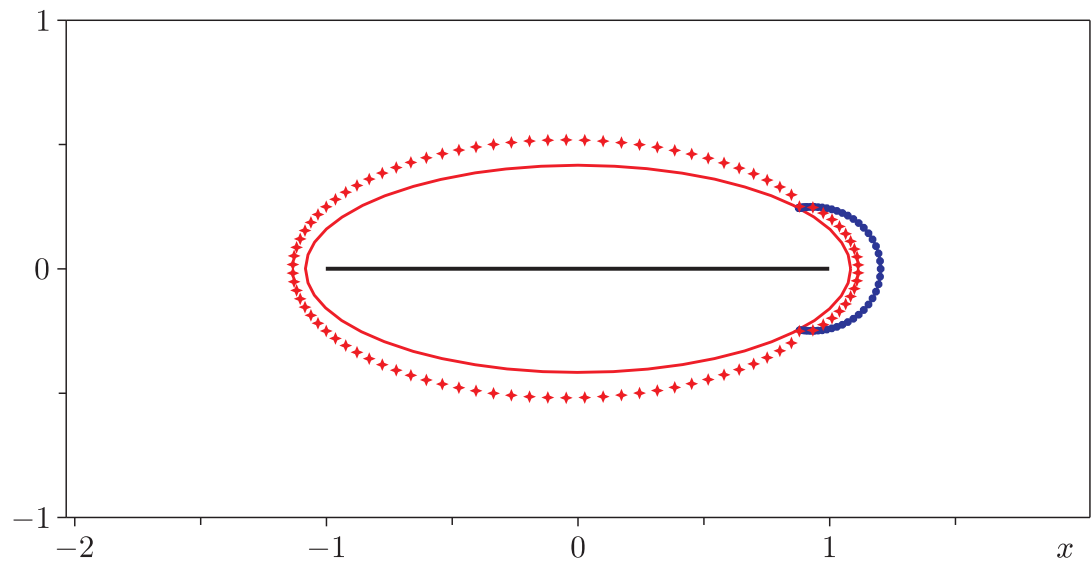

Рис. 1. Максимальный эллипс голоморфности (красная линия) функции $f(z)=\sqrt{(z-a)(z-\bar{a})}, \operatorname{Im} a>0$, и расположение нулей (красные звездочки) частичных сумм Фурье-Чебышёва $S_{100}$ и полюсов и нулей (синие точки) нелинейных аппроксимаций Паде-Чебышёва $F_{50}$ функции $f$ (см. замечание 2)

Рассмотрим теперь следующую задачу. В классе $\mathscr{R}_{n}$ найти ${ }^{2}$ рациональную функцию $F_{n}$, голоморфную на отрезке $E$ и удовлетворяющую следующему условию:

$$
c_{k}\left(F_{n}\right)=c_{k}(f), \quad k=0, \ldots, 2 n .
$$

Иными словами, разложение рациональной функции $F_{n}=P / Q$ в ряд Фурье-Чебышёва должно иметь вид

$$
F_{n}(x)=c_{0}+c_{1} T_{1}(x)+\cdots+c_{2 n} T_{2 n}+c_{2 n+1}^{*} T_{2 n+1}+\cdots .
$$

Подлежат определению из системы (11) коэффициенты многочленов $P$ и $Q$. Рациональная функция $F_{n}$ (если она существует) называется нелинейной диагональной аппроксимацией Паде-Чебышёва функции $f$ (аппроксимацией Паде ряда (10)).

Система (11) нелинейна относительно коэффициентов полиномов $P$ и $Q$ и не всегда имеет решение. Тем самым, нелинейная аппроксимация Паде-Чебышёва может не существовать. Так как полиномы Чебышёва являются полиномами Фабера для отрезка $E$, то существование нелинейной аппроксимации Паде-

\footnotetext{
${ }^{2}$ Напомним, что число свободных параметров, определяющих функцию из класса $\mathscr{R}_{n}$, равно $2 n+1$.
} 


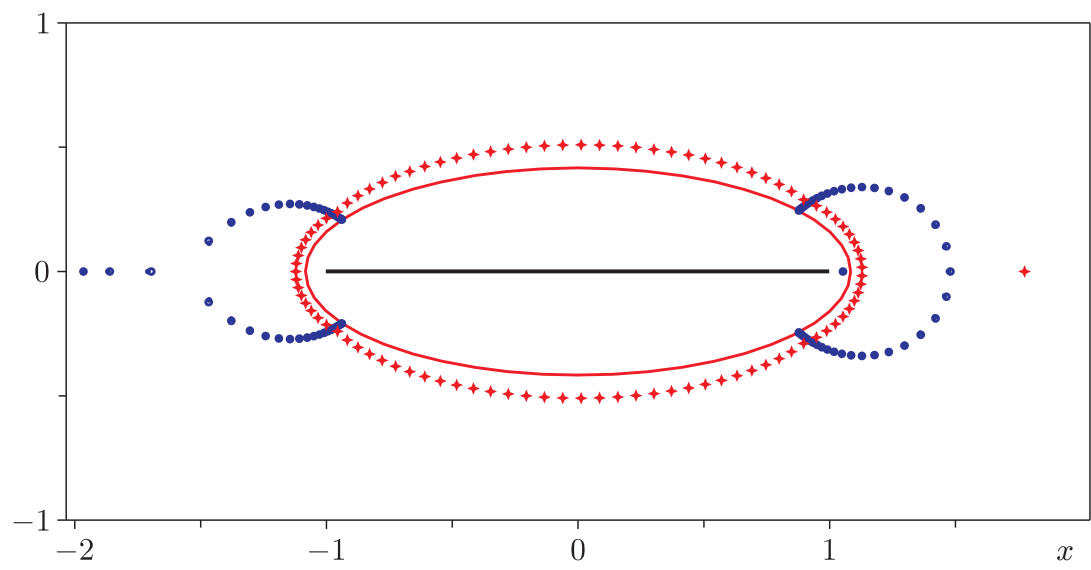

Рис. 2. Максимальный эллипс голоморфности (красная линия) функции $f(z)=\sqrt{(z-a)(z-\bar{a})}+\sqrt[3]{(z-b)(z-\bar{b})(z-c)}, \operatorname{Im} a, \operatorname{Im} b>0$, $c=-1.9$, и расположение нулей (красные звездочки) частных сумм Фурье-Чебышёва $S_{100}$ и полюсов и нулей (синие точки) нелинейных аппроксимаций Паде-Чебышёва $F_{50}$ функции $f$ (см. замечание 2 )

Чебышёва тесно связано с существованием аппроксимации Паде степенного ряда $\sum_{k=0}^{\infty} c_{k} w^{k}, c_{k}=c_{k}(f)$, обладающей определенными свойствами "регулярности" (см. прежде всего [30], а также [31], [32] и [33]). Отметим, что приведенное определение аппроксимации Паде-Чебышёва (далее - АПЧ) основано на нелинейной (относительно коэффициентов искомой рациональной функции) схеме Бейкера определения классических аппроксимаций Паде степенного ряда. Второй способ определения АПЧ, основанный на линейной схеме Фробениуса, приводит к существенно другим результатам (см. [34; ч. 2, § 1.6], а также [35]-[40]). В настоящей работе обсуждается сходимость нелинейных диагональных (типа $(n, n))$ АПЧ.

ЗАмечАниЕ 1. Представление функций в виде рядов Чебышёва активно используется в различных разделах математики при решении как теоретических, так и прикладных задач; см., например, [24], [26], [28], [29], [41]-[48]. Такое представление вполне можно рассматривать как способ эффективного задания аналитической функции; см. прежде всего монографии [49] и [28].

ЗАмЕчАНИЕ 2. Обсудим результаты вычислений, проведенных с помощью Maple и представленных на рис. 1 и 2. На обоих рисунках черная прямая линия соответствует отрезку $[-1,1]$, красная сплошная линия ограничивает максимальный эллипс голоморфности функции $f(z)$. Красная звездчатая линия соответствует нулям частичной суммы $S_{100}(z ; f)$; видно, что эти нули хорошо локализуют эллипс голоморфности $f$ - максимальную область сходимости ряда Фурье-Чебышёва (9). Синие точечные линии соответствуют нулям и полюсам нелинейной АПЧ $F_{50}$. В силу теоремы 6 эти точки локализуют $S$-компакт, соответствующий заданной функции $f$. Рациональные функции $F_{n}$ сходятся 
к функции $f$ в дополнении к этому компакту. В силу теоремы 1 аналогичный результат имеет место и для наилучших в равномерной метрике рациональных аппроксимаций $R_{n}$ для функции $f$. Однако вычисление наилучших рациональных аппроксимаций при помощи алгоритма Ремеза оказалось гораздо более трудоемкой операцией и проиллюстрировать теорему 1 на примере рациональной функции $R_{50}$ нам не удалось.

1.3. В связи с приведенной выше в п. 1.1 теоремой 1 и соотношением (8) (ср. (40)) естественным образом возникает вопрос о сходимости нелинейных АПЧ для такого же класса (см. определение 1) аналитических функций, имеющих конечное число особых точек многозначного характера. В настоящей работе доказан аналог теоремы Шталя для нелинейных АПЧ. Сделаем в этой связи два замечания.

1.3.1. В работах авторов [35] и [36] для общих ортогональных разложений были рассмотрены оба способа (Бейкера и Фробениуса) построения диагональных аппроксимаций Паде и доказаны теоремы о сходимости таких рациональных аппроксимаций для произвольной марковской функции (4) (см. ниже теорему 4). Настоящая работа является естественным развитием работ [35] и [36] для случая нелинейных АПЧ: вместо марковской функции мы рассматриваем здесь произвольную функцию, вещественную и голоморфную на $E$ и имеющую вне $E$ конечное число особых точек многозначного характера (см. определение 1). Основной результат настоящей работы - теорема 6 о скорости сходимости (по емкости) диагональных АПЧ для функций из указанного класса и о предельном распределении точек интерполяции функции $f$ рациональной функцией $F_{n}$. Эта теорема является аналогом "первой" теоремы Шталя [6] (см. также обзор [11; гл. $1, \S 3])$ о сходимости по емкости диагональных аппроксимаций Паде для функций, заданных (сходящимся) степенным рядом в точке $w=0$ и имеющих в $\overline{\mathbb{C}}_{w}$ конечное число особых точек многозначного характера (ср. теоремой 1 из п. 1.1 для наилучших диагональных аппроксимаций). При доказательстве сходимости нелинейных АПЧ мы используем эту первую теорему Шталя: благодаря результатам работы [30] о нелинейных аппроксимациях Паде-Фабера, нелинейные АПЧ функции $f$ оказываются связанными с аппроксимациями Паде степенного ряда $\sum_{k=0}^{\infty} c_{k} w^{k}, c_{k}=c_{k}(f)$, с помощью оператора Фабера-Чебышёва (подробнее см. пп. 4.1, 4.2). Тем самым, непосредственно из первой теоремы Шталя вытекает, что рациональные функции $F_{n}$ сходятся по емкости к функции $f$ в дополнении к образу компакта Шталя при отображении, задаваемом функцией Жуковского $z=\operatorname{Zh}(w):=(w+1 / w) / 2$. Таким образом, основные доказанные здесь утверждения теоремы 6 состоят в следующем:

$1)$ компакт $F$, обладающий $S$-свойством $(21)$, существует, единствен и является стационарным компактом для функционала энергии (14) при $\theta=1$; компакт $F$ совпадает с образом первого компакта Шталя $S$, заданным в плоскости переменного $w$, при отображении с помощью функции Жууковского: $F=\mathrm{Zh}(S)$;

2) справедливо соотношение (39), характеризующее скорость сходимости рациональных аппроксимаций $F_{n}$ к функции $f$ на компактных подмножествах 
области $\overline{\mathbb{C}} \backslash F$ в терминах гринова (относительно стационарного компакта $F$ ) потенциала равновесной меры $\lambda$; таким образом, для нелинейных АПЧ (той же самой функции $f \in \mathscr{A}_{E}(\overline{\mathbb{C}} \backslash \Sigma)$ ) имеет место аналог формулы (6), но с другим $S$-симметричным компактом;

3) предельное распределение точек интерполяции исходной функции $f$ рациональной функцией $F_{n}$ вполне характеризуется мерой $\lambda=\lambda^{F}$ - равновесной мерой (с носителем на $E$ ) для стационарного компакта $F$, а предельное распределение полюсов $F_{n}$ - мерой $\tilde{\lambda}=\beta_{F}(\lambda)$ - выметанием $\lambda$ из области $\overline{\mathbb{C}} \backslash F$ на $F$ (cp. (8)).

1.3.2. В [30] (см. также [31], [32]) был изучен вопрос о существовании диагональных нелинейных аппроксимаций Паде-Фабера, частным случаем которых являются аппроксимации Паде-Чебышёва. Оказалось, что при заданном номере $n$ существование такой аппроксимации тесно связано с существованием аппроксимации Паде степенного ряда $\sum_{k=0}^{\infty} c_{k} w^{k}, c_{k}=c_{k}(f)$, в смысле Бейкера и отсутствием у этой рациональной функции полюсов в замкнутом единичном круге $|w| \leqslant 1$. Эти условия выполняются, вообще говоря, не при любом $n$. Более того, в [32] приведен пример (основанный на контрпримере Буслаева [50], [51] к гипотезе Бейкера-Гаммеля-Уиллса) гиперэллиптической функции рода $g=2$, для которой нелинейные диагональные АПЧ не существуют ни при каком $n \geqslant 2$. Однако контрпример Буслаева связан с некоторым "вырождением": соответствующая гиперэллиптическая функция разлагается в периодическую непрерывную дробь. С другой стороны, из работ [52] и [53] (см. также [54]) вытекает, что в “типичной” ситуации для достаточно широкого класса аналитических функций, включающего в себя все “типичные" гиперэллиптические функции, всегда существует некоторая бесконечная подпоследовательность (зависящая от выбранной функции), для которой требуемые условия выполняются. Тем самым, для соответствующего класса многозначных функций, полученных с помощью преобразования ЖКуковского $z=\operatorname{Zh}(w)$, нелинейные АПЧ существуют по крайней мере по некоторой подпоследовательности.

1.4. Приведем необходимые определения и обозначения.

Пусть $E=[-1,1]$ - единичный отрезок на вещественной прямой $\mathbb{R}$. Через $M(E)$ обозначим множество всех единичных (положительных борелевских) мер, носители которых принадлежат $E$. Для произвольного компакта $K \subset \overline{\mathbb{C}} \backslash E$ через $D(K)$ обозначим ту связную компоненту его дополнения, которая содержит отрезок $E$; в дальнейшем будем предполагать, что область $D(K)$ регулярна относительно решения задачи Дирихле и $g_{K}(z, \zeta)$ - соответствующая области $D(K)$ функция Грина с особенностью в точке $z=\zeta \in D(K)$. Для произвольной меры $\mu \in M(E)$ определены логарифмический и гринов (по отношению к области $D(K))$ потенциалы:

$$
V^{\mu}(z)=\int_{-1}^{1} \log \frac{1}{|z-x|} d \mu(x), \quad G_{K}^{\mu}(z)=\int_{-1}^{1} g_{K}(z, x) d \mu(x), \quad z \notin E
$$

(полагаем $g_{K}(z, x) \equiv 0$ при $\left.z \in K, x \in E\right)$. Пусть $\theta \in[0, \infty)$ - произвольное фиксированное число. Для фиксированного компакта $K$ существует единственная 
мера $\lambda(\theta)=\lambda^{K}(\theta) \in M(E)$, минимизирующая функционал энергии

$$
\begin{aligned}
J_{\theta}(K ; \mu) & :=\iint_{E \times E}\left(\theta \log \frac{1}{|x-y|}+g_{K}(x, y)\right) d \mu(x) d \mu(y) \\
& =\int_{E}\left(\theta V^{\mu}(x)+G_{K}^{\mu}(x)\right) d \mu(x)
\end{aligned}
$$

в классе всех мер $\mu \in M(E)$, т. е.

$$
J_{\theta}\left(K ; \lambda^{K}\right)=\min _{\mu \in M(E)} J_{\theta}(K ; \mu) .
$$

Экстремальная мера $\lambda(\theta)$ и только эта мера (в классе $M(E))$ является равновесной мерой для смешанного (гриново-логарифмического) потенциала $\theta V^{\mu}(z)+$ $G_{K}^{\mu}(z)$. Другими словами, мера $\lambda(\theta)$ - единственная мера из класса $M(E)$, для которой на $E$ имеет место соотношение равновесия

$$
\theta V^{\lambda(\theta)}(x)+G_{K}^{\lambda(\theta)}(x) \equiv w(\theta)=\text { const }, \quad x \in E,
$$

$w(\theta)=w^{K}(\theta)$ - соответствующая постоянная равновесия; при этом $J_{\theta}(K ; \lambda)=$ $w(\theta)$ (см. [55], [56], [10]).

Введем определения и обозначения, связанные с классом аналитических функций, рассматриваемых в настоящей работе.

Пусть $\Sigma=\left\{a_{1}, \ldots, a_{p}\right\} \subset \overline{\mathbb{C}}, p \geqslant 2,-$ конечное множество, симметричное относительно вещественной прямой. В дальнейшем рассматриваются только функции $f$, голоморфные и вещественные на отрезке $E$ и допускающие аналитическое продолжение с отрезка $E$ по любому пути, лежащему в $\overline{\mathbb{C}} \backslash \Sigma$, при этом $f \notin \mathscr{H}(\overline{\mathbb{C}} \backslash \Sigma)$. Множество таких функций будем обозначать через $\mathscr{A}_{E}(\overline{\mathbb{C}} \backslash \Sigma)$. Будем также считать, что каждая точка $a_{j} \in \Sigma$ является особой точкой функции $f$ многозначного характера.

Пусть $\mathscr{K}$ - множество всех непустых компактов на плоскости $\overline{\mathbb{C}}$, а $m(K)$ число связных компонент компакта $K \in \mathscr{K}$.

Положим $\mathscr{K}^{(m)}=\{K \in \mathscr{K}: m(K) \leqslant m\}$. Тем самым, $\mathscr{K}^{(1)}$ - множество континуумов, и если $K \in \mathscr{K}^{(m)}$, то $K=\bigcup_{j=1}^{m^{\prime}} \gamma_{j}$, где $\gamma_{j} \subset \mathscr{K}^{(1)}, j=1, \ldots, m^{\prime}$, $\gamma_{s} \cap \gamma_{j}=\varnothing, s \neq j, m^{\prime} \leqslant m$; континуумы $\gamma_{j}$ будем называть компонентами $K$.

ОПредЕЛЕНИЕ 1. Пусть $\mathscr{K}_{f}$ есть множество $K \in \mathscr{K}^{(p-1)}$ таких, что:

(1) $f(z)$ допускает голоморфное (однозначное аналитическое) продолжение в область $D(K): f \in \mathscr{H}(D(K))$,

(2) любая компонента $\gamma_{j} \subset K$ содержит не менее двух точек из множества $\Sigma$. Произвольный компакт $K \in \mathscr{K}_{f}$ будем называть допустимым для заданной многозначной аналитической функции $f$ (вещественной и голоморфной на отрезке $E)$.

Если задана функция $f \in \mathscr{A}_{E}(\overline{\mathbb{C}} \backslash \Sigma)$, то тем самым зафиксирован и соответствующий ей класс допустимых компактов $\mathscr{K}_{f}$. Предполагается, что функционал энергии (14) рассматривается при произвольном $\theta \in[0, \infty)$ для этого зафиксированного класса компактов $\mathscr{K}_{f}$. 
Отметим, что с точки зрения теории рациональной аппроксимации наибольший интерес представляют значения $\theta=0,1,3$ (см. [35]-[39]).

Для заданной функции $f \in \mathscr{A}_{E}(\overline{\mathbb{C}} \backslash \Sigma)$ (см. определение 1) и параметра $\theta \in$ $[0,+\infty)$ в классе $\mathscr{K}_{f}$ допустимых компактов $K$ для $f$ рассмотрим следующую теоретико-потенциальную задачу:

$$
\sup _{K \in \mathscr{K}_{f}} \inf _{\mu \in M(E)} J_{\theta}(K ; \mu)=\sup _{K \in \mathscr{K}_{f}} J_{\theta}\left(K ; \lambda_{K}\right) .
$$

Если существует компакт $F=F(\theta) \in \mathscr{K}_{f}$, для которого достигается супремум в правой части $(16)$, то $F$ называется стационарным компактом для задачи (16) (см. определение 2). Значениям параметра $\theta=0,1,3$ соответствуют различные теоретико-потенциальные задачи (16) и, вообще говоря, различные стационарные компакты $F(0), F(1), F(3)$. Эти компакты совпадают только в исключительных случаях, например, для марковской функции $f=\widehat{\sigma}$, носитель меры которой - отрезок вещественной прямой (см. [35]-[37], а также теорему 4). При этом компакт $F(0)$ соответствует наилучшим рациональным аппроксимациям функции $f, F(1)$ - нелинейным АПЧ, $F(3)$ - линейным АПЧ, и все три стационарных (в заданном классе $\mathscr{K}_{f}$ ) компакта являются $S$-симметричными (иначе говоря, обладают $S$-свойством, см. определение 3 и [57]):

$$
\frac{\partial G_{F}^{\lambda}}{\partial n_{+}}(\zeta)=\frac{\partial G_{F}^{\lambda}}{\partial n_{-}}(\zeta), \quad \zeta \in F^{0},
$$

где $F=F(\theta), \lambda=\lambda^{F}(\theta) \in M(E)$ - соответствующая равновесная мера, $F^{0}-$ объединение всех открытых дуг, принадлежащих компакту $F, \partial / \partial n_{ \pm}-$нормальные производные, взятые с противоположных сторон $F^{0}$. Это свойство, тем самым, носит вполне универсальный характер.

ЗАмЕчАНИЕ 3. Несмотря на проблему, связанную с существованием рациональной функции $F_{n}$, в системе Maple реализованы именно нелинейные АПЧ. Такие аппроксимации активно используются в приложениях (см., например, [29], [43]-[46], [58]), они имеют преимущество перед линейными АПЧ, состоящее в том, что по заданным коэффициентам Фурье-Чебышёва $c_{0}, c_{1}, \ldots, c_{2 n}$ исходной функции $f$ можно построить нелинейную АПЧ порядка $(n, n)$, а линейную - только порядка $(m, m)$, где $m=[2 n / 3]$ (подробнее см. [35], [36]; по поводу численных методов нахождения коэффициентов Фурье-Чебышёва см., например, [26]-[28], [49]). Наилучшие равномерные рациональные аппроксимации заданной функции могут быть найдены с помощью хорошо известного алгоритма Ремеза [20]. Этот алгоритм также реализован в системе Maple. Однако процесс практического приближенного построения такой рациональной аппроксимации предполагает, что исходная функция $f$ задана в виде явного аналитического (формульного) выражения. Конечного набора коэффициентов Фурье-Чебышёва для этого недостаточно; см. замечание 2.

$\mathrm{B}$ заключение отметим, что параметрам $\theta=0, \theta=1$ и $\theta=3$ соответствуют векторные (размера $2 \times 2$ ) теоретико-потенциальные задачи равновесия. При $\theta=0$ матрица взаимодействия имеет вид $A_{0}=\left(\begin{array}{cc}1 & -1 \\ -1 & 1\end{array}\right)$, при $\theta=1-$ вид $A_{1}=\left(\begin{array}{cc}2 & -1 \\ -1 & 1\end{array}\right)$, а при $\theta=3-$ вид $A_{3}=\left(\begin{array}{cc}4 & -1 \\ -1 & 1\end{array}\right)$; cp. [59], [60]. 


\section{2. Обозначения и формулировка основных результатов}

2.1. Фиксируем произвольное $\theta \in[0, \infty)$, для этого $\theta$ рассмотрим функционал энергии (14); явное указание на параметр $\theta$ в дальнейшем иногда опускаем. Пусть $\lambda^{K} \in M(E)$ - равновесная мера с носителем на $E$, соответствующая компакту $K \in \mathscr{K}_{f}$ (см. (15)); мера $\lambda^{K}$ вполне характеризуется тем, что она является экстремальной мерой, т. е.

$$
J_{\theta}\left(K ; \lambda^{K}\right)=\min _{\mu \in M(E)} J_{\theta}(K ; \mu) .
$$

Рассмотрим следующую теоретико-потенциальную задачу:

$$
\sup _{K \in \mathscr{K}_{f}} J_{\theta}\left(K ; \lambda^{K}\right)=\sup _{K \in \mathscr{K}_{f}} \min _{\mu \in M(E)} J_{\theta}(K ; \mu) .
$$

ОПредЕЛЕНИЕ 2. Компакт $F=F(\theta) \in \mathscr{K}_{f}$ назовем стационарным (в классе $\mathscr{K}_{f}$ ) для задачи (19), если для этого компакта в (19) достигается супремум, т. е. выполняется равенство

$$
J_{\theta}\left(F ; \lambda^{F}\right)=\max _{K \in \mathscr{K}_{f}} J_{\theta}\left(K ; \lambda^{K}\right) .
$$

Вопрос о существовании и характеризации стационарных компактов (в заданном классе $\mathscr{K}_{f}$ ) представляет собой отдельную задачу. Эта задача, вообще говоря, не может быть решена непосредственно с помощью методов, разработанных в связи с проблемой модулей (см. [61]-[63]), так как заранее не известно, какому именно гомотопическому классу принадлежит такой компакт. В связи с задачей (19), связанной с конкретным функционалом энергии (14), естественным образом возникает вопрос о существовании стационарного компакта $F=F(\theta)$. Этот вопрос удается положительно решить в наиболее интересных случаях значений параметра $\theta=0,1,3$ (подробнее см. лемму 1 в п. 3.2). При этом указанным различным значениям $\theta$ соответствуют, вообще говоря, разные стационарные компакты $F(0), F(1), F(3)$ (в зафиксированном классе $\mathscr{K}_{f}$ ). В п. 2.4 приводится описание этих компактов в терминах, непосредственно связанных с соответствующими римановыми поверхностями. Отметим, что такие компакты могут совпадать при различных значениях параметра $\theta=0,1,3$ только в исключительных случаях (см. [35]-[37]).

Хорошо известно (см. [3]-[7], [10]), что с точки зрения приложения теории потенциала к исследованию сходимости рациональных аппроксимаций для многозначных аналитических функций представляет непосредственный интерес не само свойство стационарности (20), а тесно связанное с ним $S$-свойство допустимого компакта.

Приведем определение $S$-свойства допустимого компакта, соответствующего экстремальной задаче (19) для функционала энергии (14).

ОПРЕДЕЛЕНИЕ 3. Будем говорить, что не разбивающий плоскость и состоящий из конечного числа аналитических дуг допустимый компакт $\Gamma=\Gamma(\theta) \in \mathscr{K}_{f}$ обладает $S$-свойством (или является $S$-симметричным), если:

$$
\frac{\partial G_{\Gamma}^{\lambda}}{\partial n_{+}}(\zeta)=\frac{\partial G_{\Gamma}^{\lambda}}{\partial n_{-}}(\zeta), \quad \zeta \in \Gamma^{0},
$$


где $\lambda=\lambda^{\Gamma}(\theta) \in M(E)$ - соответствующая равновесная мера, $\Gamma^{0}$ - объединение всех открытых дуг, принадлежащих компакту $\Gamma, \partial / \partial n_{ \pm}-$нормальные производные, взятые с противоположных сторон $\Gamma^{0}$.

Отметим, что рассматриваемый здесь функционал энергии (14) оказывается таким, что вид соотношения (21), определяющего $S$-свойство, не зависит от параметра $\theta$. Однако равновесная мера $\lambda=\lambda^{\Gamma}(\theta) \in M(E)$ и сам $S$-симметричный компакт $\Gamma=\Gamma(\theta)$ зависят от $\theta$. Эти компакты (но не соответствующие им равновесные меры) могут совпадать только в исключительных случаях. Например, для марковской функции $f=\widehat{\sigma}$, носитель меры которой - отрезок $[c, d]$ вещественной прямой, $[c, d] \cap E=\varnothing$ :

$$
\widehat{\sigma}(z)=\int_{c}^{d} \frac{d \sigma(x)}{z-x}, \quad z \in \overline{\mathbb{C}} \backslash[c, d],
$$

мы имеем: $\Gamma(\theta)=[c, d]$ при $\theta=0,1,3$ (см. [35], [36]).

В [57] (см. также [64]) доказано, что для рассматриваемого здесь функционала энергии (14) и класса $\mathscr{K}_{f}$ допустимых компактов (см. определение 1 ) введенные понятия оказываются равносильными: стационарный компакт $F=$ $F(\theta) \in \mathscr{K}_{f}$ (если он существует) всегда обладает $S$-свойством (21). При этом для $\theta=0,1,3$ он существует (см. лемму 1 в п. 3.2), является единственным и указанное $S$-свойство его вполне характеризует; тем самым, $F(\theta)=\Gamma(\theta)$. Обозначение $\Gamma(\theta)$ далее в настоящей работе не используется.

Справедлива следующая теорема (см. [57]).

ТЕОрема 2. Пусть $F=F(\theta) \in \mathscr{K}_{f}$ - стационарный компакт для задачи (19) (состоящий из конечного числа континуумов). Тогда $F$ не разбивает плоскость, не имеет внутренних точек и обладает $S$-свойством (21).

Подчеркнем, что условие $F \in \mathscr{K}_{f}$ предполагает, что компакт $F$ состоит из конечного числа континуумов. В дальнейшем нам удобно ввести обозначение для числа $p$ точек ветвления: $p=m-2$ и считать, что все $a_{j} \neq \infty$.

Теорема 2 является следствием следующего утверждения.

Теорема 3. Пусть $F \in \mathscr{K}_{f}$ - стационарный компакт для задачи (19) и $A_{m}(z)=\left(z^{2}-1\right)\left(z-a_{1}\right) \cdots\left(z-a_{m-2}\right)$, где $\left\{a_{1}, \ldots, a_{m-2}\right\}=\Sigma$ и все $a_{j}$ конечнъь. Тогда для равновесной мерь $\lambda=\lambda^{F}$ компакта $F$ справедливо следующее соотношение (необходимое условие стационарности):

$$
\theta \widehat{\lambda}(z)^{2}+\left(\mathscr{P}_{F, \lambda}^{\prime}(z)\right)^{2} \equiv \frac{B_{m-1}(z)}{A_{m}(z)}, \quad z \in D(F) \backslash E,
$$

где $B_{m-1}(z)$ - некоторый полином степени $\leqslant m-1, \widehat{\lambda}(z)=\int(z-x)^{-1} d \lambda(x)-$ преобразование Коши меры $\lambda$,

$$
\mathscr{P}_{F, \lambda}(z)=\int_{-1}^{1} P(z, x) d \lambda(x)
$$

$P(z, x)=g_{F}(z, x)+i g_{F}^{*}(z, x)-$ комплексная функция Грина для области $D(F)$. 
Отметим, что в силу равенства

$$
\widehat{\lambda}(z)=-\mathscr{V}_{\lambda}^{\prime}(z), \quad \text { где } \mathscr{V}_{\lambda}(z)=\int_{-1}^{1} \log \frac{1}{z-x} d \lambda(x),
$$

соотношение (23) можно представить в виде

$$
\theta\left(\mathscr{V}_{\lambda}^{\prime}(z)\right)^{2}+\left(\mathscr{P}_{F, \lambda}^{\prime}(z)\right)^{2} \equiv \frac{B_{m-1}(z)}{A_{m}(z)} .
$$

Следующий результат вытекает непосредственно из теоремы 3 (cp. [3], [4], [10], [65; §4, п. 4.3, теорема XII]), [66].

ПРЕДЛОЖЕНИЕ 1. При $\theta=0$ справедливо представление

$$
G_{F}^{\lambda}(z)=\int_{-1}^{1} g_{F}(z, x) d \lambda(x)=\operatorname{Re} \int_{a_{1}}^{z} \sqrt{\frac{B_{m-1}(\zeta)}{A_{m}(\zeta)}} d \zeta, \quad z \in \overline{\mathbb{C}} \backslash(E \cup F) ;
$$

соотношение (25) позволяет однозначно определить равновесную меру $\lambda u$ стационарный компакт $F$, если известен полином $B_{m-1}$.

Оказывается, что теоретико-потенциальную задачу (19) можно переформулировать эквивалентным образом в других терминах (см. [38], [57], [67] и п. 3.1 ниже для $\theta=1)$. Зафиксируем параметр $\theta \in[0, \infty)$ и класс допустимых компактов $\mathscr{K}_{f}$. При условии, что $D(K) \ni \infty$, экстремальная задача (18) в классе единичных мер $M(E)$ с носителями на $E$ эквивалентна следующей экстремальной задаче в классе единичных мер $M(K)$ с носителями на $K$ :

$$
\inf _{\nu \in M(K)} I_{\varphi}(K ; \nu)
$$

где

$$
\begin{aligned}
I_{\varphi}(K ; \nu) & :=\iint_{K \times K}\left\{\theta \log \frac{1}{|z-\zeta|}+g_{E}(z, \zeta)+\theta g(z, \infty)+\theta g(\zeta, \infty)\right\} d \nu(z) d \nu(\zeta) \\
& =\int_{K}\left\{\theta V^{\nu}(z)+G_{E}^{\nu}(z)\right\} d \nu(z)+2 \int_{K} \theta g_{E}(z, \infty) d \nu(z)
\end{aligned}
$$

$g_{E}(z, \zeta)$ - функция Грина для дополнения к отрезку $E$ с особенностью в точке $z=\zeta$

$$
G_{E}^{\nu}(z):=\int_{K} g_{E}(z, \zeta) d \nu(\zeta)
$$

- гринов (относительно $\overline{\mathbb{C}} \backslash E$ ) потенциал меры $\nu \in M(K)$. Иначе говоря, $I_{\varphi}(K ; \nu)$ - энергия меры $\nu$ относительно смешанного гриново-логарифмического ядра $-\theta \log |z-\zeta|+g_{E}(z, \zeta)$ во внешнем поле $\varphi(z):=\theta g(z, \infty)$. Пусть $\lambda_{K} \in M(K)$ - соответствующая задаче (26) экстремальная мера:

$$
I_{\varphi}\left(K ; \lambda_{K}\right)=\min _{\nu \in M(K)} I_{\varphi}(K ; \nu)
$$

Тогда эта же мера $\lambda=\lambda_{K}$ является (единственной) равновесной мерой для потенциала $\theta V^{\nu}+G_{E}^{\nu}$ во внешнем поле $\varphi$ :

$$
\theta V^{\nu}(z)+G_{E}^{\nu}(z)+\varphi(z) \equiv w_{K}, \quad z \in K,
$$

$w_{K}$ - соответствующая постоянная равновесия. 
Можно показать (см. [67] и ниже п. 3.1 для случая $\theta=1$ ), что для равновесных мер $\lambda^{K}$ и $\lambda_{K}$, соответствующих функционалам энергии (14) и (27), справедливо соотношение

$$
I_{\varphi}\left(K ; \lambda_{K}\right)=(1+\theta) J_{\theta}\left(K ; \lambda^{K}\right)-\theta^{2} \gamma_{E},
$$

где $\gamma_{E}=2 \log 2-$ постоянная Робена для отрезка $E=[-1,1]$. Непосредственно из (31) вытекает, что при фиксированном компакте $E$ (в нашем случае $E=$ $[-1,1])$ две экстремальные задачи

$$
\sup _{K \in \mathscr{K}_{f}} J_{\theta}\left(K ; \lambda^{K}\right)=\sup _{K \in \mathscr{K}_{f}} \inf _{\mu \in M(E)} J_{\theta}(K ; \mu)
$$

и

$$
\sup _{K \in \mathscr{K}_{f}} I_{\varphi}\left(K ; \lambda_{K}\right)=\sup _{K \in \mathscr{K}_{f}} \inf _{\nu \in M(K)} I_{\varphi}(K ; \nu)
$$

эквивалентны, а сами равновесные меры $\lambda^{K} \in M(E)$ и $\lambda_{K} \in M(K)$ для компакта $K$ связаны соотношением: $\lambda_{K}=\beta_{K}\left(\lambda^{K}\right)$, где $\beta_{K}(\cdot)$ - выметание меры из области $D(K)$ на ее границу.

В этой эквивалентной постановке задача (32) допускает такую геометрическую интерпретацию, которая позволяет сделать следующий вывод: при $\theta=0,1,3$ стационарный компакт $F \in \mathscr{K}_{f}$ существует и состоит из конечного числа континуумов. Тем самым, в этой ситуации применима теорема 2 и оказывается, что стационарный компакт всегда обладает $S$-свойством.

ЗАмечАнИЕ 4. Вопрос о единственности компакта $F=F(\theta) \in \mathscr{K}_{f}$ решается следующим образом. При $\theta=0,1,3$ теоретико-потенциальная задача (19) оказывается связанной с задачей о сходимости соответствующих "оптимальных" рациональных аппроксимаций $f_{n}(z ; \theta)$ функции $f(z)$. На основе $S$-свойства стационарного компакта стандартным методом (см. [10], а также [37]-[39], [68]) устанавливается, что $f_{n}(z ; \theta) \stackrel{\text { сар }}{\longrightarrow} f(z)$ в области $D_{f}=D(F(\theta)):=$ $\overline{\mathbb{C}} \backslash F(\theta)$ (здесь и ниже " $\stackrel{\text { сар }}{\longrightarrow}$ " означает сходимость по логарифмической емкости на компактных подмножествах области $D_{f}$; определение этого понятия см., например, в [6], [7], [9]). Непосредственно отсюда и следует единственность компакта $F(\theta), \theta=0,1,3$. В каждом из этих трех случаев для равновесных мер $\lambda^{F}(\theta) \in M(E)$ и $\lambda_{F}(\theta) \in M(F)$, соответствующих (единственному) стационарному компакту $F$, справедливы соотношения

$$
\frac{1}{n} \mu\left(Q_{n}\right) \rightarrow \lambda_{F}, \quad \frac{1}{2 n} \mu\left(\omega_{2 n}\right) \rightarrow \lambda^{F} \quad \text { при } n \rightarrow \infty,
$$

где $Q_{n}, \operatorname{deg} Q_{n} \leqslant n,-$ знаменатель рациональной дроби $f_{n}$, а $\omega_{2 n}, \operatorname{deg} \omega_{2 n} \leqslant$ $2 n+1,-$ полином, в нулях ${ }^{3}$ которого эта рациональная дробь интерполирует исходную функцию $f$. Скорость сходимости рациональных функций $f_{n}(z ; \theta)$ к $f(z)$ в области $D_{f}$ задается функцией $\exp \left\{-G_{F}^{\lambda^{F}}(z)\right\}<1, z \in D_{f}$.

Таким образом, реализуется следующая стандартная схема рассуждений, предложенная в [10]: существует стационарный компакт $F$ для функционала энергии $(14) \Rightarrow$ компакт $F$ обладает $S$-свойством $(21)$ (тем самым, в допустимом

\footnotetext{
${ }^{3}$ Все они лежат на $E$.
} 
классе $\mathscr{K}_{f}$ существует $S$-компакт $\left.\Gamma=F\right) \Rightarrow f_{n} \stackrel{\text { сар }}{\longrightarrow} f$ в "максимальной" области голоморфности $D_{f}:=\overline{\mathbb{C}} \backslash F \Rightarrow S$-компакт единственный. Максимальность области голоморфности $D_{f}$ функции $f$ понимается так: дополнение $F$ к ней минимально в том смысле, что величина $e^{-w^{K}}$, которую естественно считать аналогом емкости, минимальна для $K=F, K \in \mathscr{K}_{f}$.

ЗАмЕчаниЕ 5. Все утверждения теорем 2, 3 сохраняются, если при определении класса функций $\mathscr{A}_{E}(\overline{\mathbb{C}} \backslash \Sigma)$ вместо голоморфных функций допустить мероморфные функции.

Таким образом, при условии существования экстремального компакта, решающего задачу (19), все его дальнейшие свойства устанавливаются общим вариационным методом, предложенным в [66] (см. также [57]) и применимым при произвольном $\theta \in[0, \infty)$ (см. пп. 3.1-3.3).

2.2. В [35] и [36] была изучена сходимость нелинейных аппроксимаций $F_{n}$ и аппроксимаций Фробениуса $\Phi_{n}$ для общих ортогональных разложений марковских функций

$$
\widehat{\sigma}(z)=\int_{F} \frac{d \sigma(x)}{z-x}, \quad z \in \overline{\mathbb{C}} \backslash F,
$$

где $F=[c, d] \subset \mathbb{R} \backslash E, \sigma$ - положительная борелевская мера на $F, \sigma^{\prime}=$ $d \sigma / d x>0$ почти всюду (п. в.) на $F$. Скорость сходимости последовательностей $R_{n}, F_{n}$ и $\Phi_{n}$ к функции $f=\widehat{\sigma}$ в области $D_{f}=\overline{\mathbb{C}} \backslash[c, d]$ полностью характеризуется в терминах равновесной меры $\lambda(\theta) \in M(E)$ соответственно для $\theta=0, \theta=1$ и $\theta=3$ следующим образом (см. [2], [35], [36]).

ТеОрема 4. Если $\sigma^{\prime}>0$ n.в. на $F=[c, d] \subset \mathbb{R} \backslash E$, то локально равномерно в области $D_{f} \backslash E$

$$
\lim _{n \rightarrow \infty}\left|\left(\widehat{\sigma}-f_{n}\right)(z)\right|^{1 / n}=e^{-2 G_{F}^{\lambda(\theta)}(z)}<1,
$$

где $\theta=0$ для $f_{n}=R_{n}, \theta=1$ для $f_{n}=F_{n} u \theta=3$ для $f_{n}=\Phi_{n}$.

Обозначим через $\mu(Q)$ меру, ассоциированную с произвольным полиномом $Q: \mu(Q)=\sum_{\zeta: Q(\zeta)=0} \delta_{\zeta}$, где $\delta_{\zeta}-$ мера Дирака с носителем в точке $\zeta$. Пусть $\widetilde{\mu}$ - выметание меры $\mu$ из области $\overline{\mathbb{C}} \backslash F$ на $F$. Тогда в условиях теоремы 4 для знаменателей $Q_{n}(z ; \theta), \quad \theta=0,1,3$, соответствующих рациональных функций $R_{n}, F_{n}, \Phi_{n}$ имеем:

$$
\frac{1}{n} \mu\left(Q_{n}(\cdot ; \theta)\right) \rightarrow \widetilde{\lambda}(\theta), \quad n \rightarrow \infty
$$

где сходимость мер понимается в слабой топологии.

Отметим, что развитые в [2], [35], [36] методы позволяют легко доказать аналог теоремы 4 и для случая, когда $F$ состоит из нескольких отрезков, лежащих на вещественной прямой, а $f=\widehat{\sigma}+r$, где $\widehat{\sigma}$ - марковская функция (35), $r$ вещественная рациональная функция, голоморфная на $E$. 
2.3. Зафиксируем теперь параметр $\theta=1$ и в дальнейшем в обозначениях будем, как правило, опускать указание на этот параметр. Пусть $\lambda=\lambda^{K}(1) \in$ $M(E)$ - равновесная мера, соответствующая произвольному компакту $K \in \mathscr{K}_{f}$, а $w=w^{K}(1)$ - соответствующая постоянная равновесия (см. (15)): $V^{\lambda}(x)+$ $G_{K}^{\lambda}(x) \equiv w, x \in E ;$ при этом $J(K ; \lambda)=\min _{\mu \in M(E)} J(K ; \mu)=w$.

Справедлива следующая теорема.

Теорема 5. Если $f \in \mathscr{A}_{E}(\overline{\mathbb{C}} \backslash \Sigma)$, то существует единственный компакт $F=F(1) \in \mathscr{K}_{f}$ такой, что

$$
J\left(F ; \lambda^{F}\right)=\max _{K \in \mathscr{K}_{f}} J\left(K ; \lambda^{K}\right) .
$$

Стационарный компакт $F$ состоит из конечного числа аналитических дуг, не разбивает плоскость и обладает $S$-свойством (21), где $\lambda^{F}=\lambda^{F}(1)$ - coответствующая равновесная мера.

Теорема 5 вытекает непосредственно из теоремы 2 и леммы 1 (см. п. 3.2). Фактически доказательство теоремы 5 проводится по следующей схеме. Сначала используется соотношение (31), устанавливающее связь между функционалами энергии (14) и (27). Затем с помощью этого соотношения и стандартных геометрических соображений, основанных на замене функционала энергии (27) для равновесной меры $\lambda \in M(K)$ на обобщенный трансфинитный диаметр $K \in \mathscr{K}_{f}$ (см. [55; гл. II, §3]), устанавливается, что (вообще говоря, локальный) супремум в правой части (33) можно рассматривать среди тех допустимых компактов, которые лежат во внешности максимального канонического эллипса голоморфности функции $f$. Такое семейство предкомпактно в хаусдорфовой топологии, поэтому существует допустимый компакт $F, F \subset G_{0}^{c}(f)$, удовлетворяющий соотношению (38). Затем с помощью вариационного метода (см. [57], [66], [69], [70]) устанавливается, что этот экстремальный компакт $F$ является замыканием критических траекторий некоторого квадратичного дифференциала. Непосредственно отсюда уже вытекает $S$-свойство $(21)$ (для $\theta=1$ ).

На основе теоремы 5 доказывается следующая теорема о скорости сходимости рациональных аппроксимаций $F_{n}$ к функции $f$ в области $D_{f}=D(F)=\overline{\mathbb{C}} \backslash F$ (ср. с теоремой 1 и соотношением (6)).

Tеорема 6. Пусть $f \in \mathscr{A}_{E}(\overline{\mathbb{C}} \backslash \Sigma)$. Тогда для любого компакта $K \subset \overline{\mathbb{C}} \backslash$ $(E \cup F)$ имеем:

$$
\left|\left(f-F_{n}\right)(z)\right|^{1 / n} \stackrel{\text { cap }}{\longrightarrow} e^{-2 G_{F}^{\lambda}(z)}<1, \quad z \in K,
$$

где $\lambda=\lambda^{F}, F=F(1)$. При этом для точек интерполяции функции $f$ рациональной функиией $F_{n}$ на отрезке $E$ и нулей знаменателя $Q_{n}$ выполняются соотношения

$$
\frac{1}{2 n} \mu\left(\omega_{2 n}\right) \rightarrow \lambda, \quad \frac{1}{n} \mu\left(Q_{n}\right) \rightarrow \widetilde{\lambda}, \quad n \rightarrow \infty
$$

где $\omega_{2 n}$ - полином степени $2 n+1$ с нулями в точках интерполяции, $\tilde{\lambda}=$ $\beta_{F}(\lambda)$ - выметание мерь $\lambda \in M(E)$ из области $D_{f}$ на $\partial D_{f}=F$. 
Тем самым, в условиях теоремы $6 F_{n} \stackrel{\text { сар }}{\longrightarrow} f$ на компактных подмножествах области $D_{f}=\overline{\mathbb{C}} \backslash F$ со скоростью геометрической прогрессии.

Теорема 6 доказывается по общей схеме, предложенной в [3]-[7] и [10] и основанной на $S$-свойстве $(21)$ соответствующего стационарного компакта. При этом используется теорема Шталя о сходимости классических аппроксимаций Паде степенного ряда. При доказательстве предельных соотношений (40) используется общая теоремы 3 работы [10] (для рассматриваемого здесь частного случая внешнего поля $\left.\psi(z)=-V^{\lambda}(z)\right)$. Отметим, что из (39) вытекает, что каждый полюс $f$ в $D_{f}$ притягивает по меньшей мере столько полюсов $f_{n}$, какова его кратность.

Таким образом, для функции $f$ из класса $\mathscr{A}_{E}(\overline{\mathbb{C}} \backslash \Sigma)$ компакт $F(1)$ в случае нелинейных АПЧ играет роль отрезка $[c, d]$, соответствующего марковской функции $\widehat{\sigma}, \operatorname{supp} \sigma=[c, d]($ см. (35)).

2.4. Если (для параметра $\theta=1$ ) стационарный компакт $F=F(1)$, соответствующий функции $f \in \mathscr{A}_{E}(\overline{\mathbb{C}} \backslash \Sigma)$, состоит из $s$ непересекающихся аналитических дуг, попарно соединяющих некоторые точки ветвления $a_{1}^{\prime}, \ldots, a_{2 s}^{\prime} \in \Sigma$ функции $f$, то он допускает наглядное описание в терминах, связанных с четырехлистной римановой поверхностью рода $g=s-1$ (ср. [57; §4].

Построим сначала двулистную риманову поверхность $\mathfrak{R}=\mathfrak{R}^{(1)} \cup \mathfrak{R}^{(2)}$ следующим образом. Возьмем два экземпляра римановой сферы $\overline{\mathbb{C}}$, разрезанных по отрезку $E=[-1,1]$, и переклеим по разрезу. Полученная двулистная риманова поверхность $\mathfrak{R}=\mathfrak{R}^{(1)} \cup \mathfrak{R}^{(2)}$ эквивалентна римановой сфере. Определим на $\mathfrak{R}$ функцию $u(\mathbf{z}), \mathbf{z} \in \mathfrak{R}$, следующим образом: $u\left(z^{(1)}\right)=G_{F}^{\lambda}(z)$, $u\left(z^{(2)}\right)=w^{F}-V^{\lambda}(z)$. Непосредственно из условия равновесия (15) вытекает, что $u$ - гармоническая функция на $\mathfrak{R} \backslash\left(F^{(1)} \backslash\left\{\infty^{(2)}\right\}\right)$. Кроме того, $u \equiv 0$ на компакте $F^{(1)}$ и $u\left(z^{(2)}\right)=\log |z|+w^{F}+o(1)$ при $z^{(2)} \rightarrow \infty^{(2)}$. Возьмем теперь два экземпляра $\mathfrak{R}$, разрезанных по компакту $F^{(1)}$, и переклеим их между собой по соответствующим разрезам. Получим четырехлистную риманову поверхность $\mathfrak{R}_{1}$ рода $g=s-1$. Так как $u \equiv 0$ на $F^{(1)}$, то $u$ гармонически продолжается с одного экземпляра $\mathfrak{R}$ на другой с переменой знака. Продолженная функция гармонична на $\mathfrak{R}_{1}$ всюду кроме точек $\mathbf{z}=\infty^{(2)}, \infty^{(3)}$, где она имеет логарифмические особенности: $\log |z|$ при $\mathbf{z} \rightarrow \infty^{(2)}$ и $-\log |z|$ при $\mathbf{z} \rightarrow \infty^{(3)}$. Следовательно, $u(\mathbf{z})=\operatorname{Re} \Omega(\mathbf{z}), \Omega(\mathbf{z})=\int_{b_{1}}^{\mathbf{z}} d \Omega$, где $d \Omega(\mathbf{z})-($ единственный) абелев дифференциал на $\mathfrak{R}_{1}$ с чисто мнимыми периодами и особенностью вида $1 / z$ в точке $\mathbf{z}=\infty^{(2)}$ и вида $-1 / z$ в точке $\mathbf{z}=\infty^{(3)}$. Компакт $F$ соответствует нулевой линии уровня функции $\operatorname{Re} \Omega(\mathbf{z}): F=\{z \in \overline{\mathbb{C}}: \operatorname{Re} \Omega(\mathbf{z})=0\} \backslash E$.

Отметим, что $u(\mathbf{z})=g_{F^{(1)}}\left(\mathbf{z}, \infty^{(2)}\right)-$ функция Грина для области $\mathfrak{R} \backslash F^{(1)}$ с особенностью в точке $\mathbf{z}=\infty^{(2)}$, а $w^{F}=\gamma^{(2)}$ - постоянная Робена для этой функции Грина. Тем самым, задаче о максимуме постоянной $w^{F}$ соответствует задача о минимуме $e^{-\gamma^{(2)}}$, т. е. минимуме логарифмической емкости.

Непосредственно из результатов работ [3]-[7] и [10] вытекает, что для наилучuиx в равномерной метрике на отрезке $[-1,1]$ рациональных аппроксимаций $f_{n}=R_{n}$ функции $f \in \mathscr{A}_{E}(\overline{\mathbb{C}} \backslash \Sigma)$ также справедливо соотношение вида (39), где $\theta=0, F=F(0)$ - стационарный компакт, соответствующий задаче равновесия (15) с $\theta=0$ и обладающий $S$-свойством $(21), \lambda=\lambda^{F}(0)$ - соответствующая 
равновесная мера (см. теорему 1). В этом случае функция $u\left(z^{(1)}\right)=w^{F}-G^{\lambda}(z)$ продолжается через разрез, проведенный по отрезку $E$, на второй лист римановой поверхности $\mathfrak{R}=\mathfrak{R}^{(1)} \cup \mathfrak{R}^{(2)}$ с переменой знака. Отсюда уже легко вытекает, что задача о максимуме постоянной $w^{F}$ эквивалентна задаче о минимуме емкости конденсатора $\left(F^{(1)}, F^{(2)}\right)$.

Таким образом, оба стационарных (в заданном классе $\mathscr{K}_{f}$ ) компакта $F(1)$ и $F(0)$ обладают $S$-свойством вида $(21)$ (соответственно для $\theta=1$ и $\theta=0$ ).

\section{3. Доказательство теоремы 5}

3.1. Пусть $K \in \mathscr{K}_{f}$ - произвольный допустимый компакт для функции $f \in \mathscr{A}_{E}(\overline{\mathbb{C}} \backslash \Sigma)$ и $D(K)$ - связная компонента его дополнения, которая содержит отрезок $E$; мы предполагаем, что область $D(K)$ регулярна относительно решения задачи Дирихле. В дальнейшем мы предполагаем также, что ${ }^{4} \infty \in D(K)$, $\infty \notin \Sigma$.

Пусть $\lambda=\lambda^{K} \in M(E)$ - равновесная мера для $K$ :

$$
V^{\lambda}(x)+G_{K}^{\lambda}(x) \equiv w^{K}, \quad x \in E,
$$

величина $w=w^{K}$ - "постоянная Робена" для $K, e^{-w}$ - "емкость" компакта $K$ (соответствующая энергии взаимодействия $J(\cdot)$; см. (14)). Пусть $\widetilde{\lambda}=\beta_{K}(\lambda)$ выметание равновесной меры $\lambda$ на $K$. Рассмотрим функцию

$$
v(z):=V^{\lambda}(z)+G_{K}^{\lambda}(z)+G_{E}^{\tilde{\lambda}}(z)+g_{E}(z, \infty),
$$

где $G_{E}^{\nu}(z)$ - гринов относительно $E$ потенциал меры $\nu$,

$$
G_{E}^{\nu}(z)=\int g_{E}(z, \zeta) d \nu(\zeta), \quad \operatorname{supp} \nu \subset \overline{\mathbb{C}} \backslash E,
$$

$g_{E}(z, \zeta)$ - функция Грина для дополнения к отрезку $E, g_{E}(z, \infty)=\log \mid z+$ $\sqrt{z^{2}-1} \mid$. Нетрудно видеть, что сумма $G_{K}^{\lambda}(z)+G_{E}^{\tilde{\lambda}}(z)-$ функция, гармоническая вне $E$. Из (42) получаем, что и функция $v(z)$ гармонична в $\overline{\mathbb{C}} \backslash E$. Из соотношения равновесия (41) вытекает, что $v(x) \equiv w^{K}$ при $x \in E$. Следовательно, $v(z) \equiv w^{K}$ при $z \in \overline{\mathbb{C}}$. Отсюда для $z \in K$ получаем

$$
w^{K} \equiv v(z)=V^{\lambda}(z)+G_{E}^{\tilde{\lambda}}(z)+g_{E}(z, \infty), \quad z \in K .
$$

Но при $z \in K$ потенциал $V^{\lambda}(z)$ равен $V^{\tilde{\lambda}}(z)-c_{K}$, где $c_{K}=\int_{E} g_{K}(x, \infty) d \lambda^{K}(x)$. Таким образом, из (43) получаем, что для выметания $\widetilde{\lambda} \in M(K)$ равновесной меры $\lambda \in M(E)$ справедливо равенство:

$$
V^{\tilde{\lambda}}(z)+G_{E}^{\tilde{\lambda}}+g_{E}(z, \infty) \equiv \mathrm{const}=w^{K}+c_{K}=: w_{K}, \quad z \in K .
$$

\footnotetext{
$4 \ni$ ти условия $D(K) \ni \infty$ и $\Sigma \not \supset \infty$ не являются ограничительными и носят чисто технический характер: их выполнения всегда можно добиться с помощью подходящего дробно-линейного преобразования, сохраняющего отрезок $E$ (пока речь идет не об аппроксимации ряда (9) рациональными функциями, а о теоретико-потенциальных задачах равновесия (47) и (48)).
} 
Следовательно, мера $\widetilde{\lambda} \in M(K)$ является (единственной) равновесной мерой в классе $M(K)$ для потенциала $V^{\nu}(z)+G_{E}^{\nu}(z)$ с внешним полем $\varphi(z)=g_{E}(z, \infty)$. Таким образом, отображение $\lambda \mapsto \widetilde{\lambda}$ задает взаимно однозначное соответствие между равновесными мерами для задач (41) и (44). Хорошо известно (см. [10], [55], [56], [71]), что равновесная мера и только эта мера в классе мер $\nu \in M(K)$ минимизирует соответствующий функционал энергии

$$
\begin{aligned}
I_{\varphi}(K ; \nu) & :=\iint\left\{\log \frac{1}{|z-\zeta|}+g_{E}(z, \zeta)\right\} d \nu(z) d \nu(\zeta)+2 \int \varphi(z) d \nu(z) \\
& =\int\left\{V^{\nu}(z)+G_{E}^{\nu}(z)+\varphi(z)\right\} d \nu(z)+\int \varphi(z) d \nu(z)=w_{K}+c_{E} .
\end{aligned}
$$

При этом для соответствующих задачам (41) и (44) постоянных равновесия имеем: $w^{K}=w_{K}-c_{K}$. Так как $v(z) \equiv w^{K}$, то из (42) получаем, что $w^{K}=$ $v(\infty)=\gamma_{E}+c_{K}+c_{E}$, где

$$
c_{K}=\int_{E} g_{K}(x, \infty) d \lambda(x), \quad c_{E}=\int_{K} g_{E}(\zeta, \infty) \tilde{d}(\zeta),
$$

$\gamma_{E}=2 \log 2$ - постоянная Робена для $E$. Отсюда с учетом (44) и (45) уже легко получаем, что соответствующие равновесным мерам $\lambda$ и $\tilde{\lambda}$ энергии связаны соотношением

$$
I_{\varphi}(K ; \widetilde{\lambda})=2 J(K ; \lambda)-\gamma_{E}
$$

Непосредственно из (46) вытекает, что задача

$$
\sup _{K \in \mathscr{K}_{f}} \inf _{\mu \in M(E)} J(K ; \mu)
$$

эквивалентна (в предположении, что существует экстремальный компакт $F \in$ $\mathscr{K}_{f}$ такой, что $\left.D(F) \ni \infty\right)$ задаче

$$
M=\sup _{K \in \mathscr{K}_{f}} \inf _{\nu \in M(K)} I_{\varphi}(K ; \nu), \quad \text { где } \varphi(z)=g_{E}(z, \infty) .
$$

Ниже мы докажем существование стационарного компакта $F$ для задачи (48). В силу указанной эквивалентности это будет означать, что этот же компакт $F$ является стационарным компактом и для задачи (47) в предположении, что $D(F) \ni \infty$. Единственность такого компакта будет вытекать, как обычно, из сходимости АПЧ к функции $f \in \mathscr{H}(D(F))$. Условия $F \not \supset \propto$ и $\Sigma \not \supset \propto$ не являются ограничительными и носят чисто технический характер: их всегда можно добиться с помощью подходящего дробно-линейного преобразования, сохраняющего отрезок $E$ (пока речь идет не об аппроксимации ряда (9) рациональными функциями, а о теоретико-потенциальных задачах равновесия (47) и (48)). Отметим, что непосредственно для исследования сходимости аппроксимаций нужно эквивалентное стационарности свойство $S$-симметрии компакта $F$. Как следует из определения (см. (21)), это свойство инвариантно относительно дробно-линейных преобразований и вообще не зависит от того, содержит компакт $F$ точку $z=\infty$ или нет. 
В заключение этого пункта приведем еще одну эквивалентную переформулировку экстремальной скалярной задачи (47) в виде матричной (размера $2 \times 2$ ) теоретико-потенциальной задачи равновесия (см. [10], [59]). Рассмотрим следующую векторную задачу равновесия в классе вектор-мер $\vec{\mu}=\left(\mu_{1}, \mu_{2}\right)$, $\mu_{1} \in M(E), \mu_{2} \in M(K), K \in \mathscr{K}_{f}:$

$$
\begin{aligned}
2 V^{\mu_{1}}(x)-V^{\mu_{2}}(x) & \equiv w_{1}, \quad x \in E, \\
-V^{\mu_{1}}(z)+V^{\mu_{2}}(z) \equiv w_{2}, & z \in K,
\end{aligned}
$$

с матрицей взаимодействия $A=\left(\begin{array}{cc}2 & -1 \\ -1 & 1\end{array}\right)$. Векторная задача (49) эквивалентна скалярной задаче

$$
V^{\mu}(x)+G_{K}^{\mu}(x) \equiv w, \quad x \in E, \quad \mu \in M(E) .
$$

При этом решение $\vec{\lambda}=\left(\lambda_{1}, \lambda_{2}\right)$ задачи (49) связано с решением задачи (50) следующим образом: $\lambda_{1}=\lambda, \lambda_{2}=\widetilde{\lambda}-$ выметание меры $\lambda \in M(E)$ из $\overline{\mathbb{C}} \backslash K$ на $K$, а для соответствующих постоянных равновесия $w_{1}, w_{2}$ и $w$ имеем: $w_{2}=$ $c_{K}(\lambda)=\int_{E} g_{K}(x, \infty) d \lambda(x), w_{1}=w-w_{2}$; тем самым, $w=w_{1}+w_{2}$. Таким образом, скалярная экстремальная задача (47) может быть эквивалентным образом переформулирована в виде следующей векторной задачи (ср. [59], [60], [72]):

$$
\sup _{K \in \mathscr{K}_{f}} \inf _{\substack{\left(\mu_{1}, \mu_{2}\right), \mu_{1} \in M(E), \mu_{2} \in M(K)}} J_{A}(K ; \vec{\mu}),
$$

где

$$
\begin{aligned}
J_{A}(K ; \vec{\mu}) & :=(A \vec{\mu}, \vec{\mu})=\sum_{k, j=1}^{2} a_{k, j}\left[\mu_{k}, \mu_{j}\right], \\
{\left[\mu_{k}, \mu_{j}\right] } & =\iint_{K \times K} \log \frac{1}{|z-\zeta|} d \mu_{k}(z) d \mu_{j}(\zeta) .
\end{aligned}
$$

3.2. Этот и следующий пункты посвящены доказательству существования стационарного компакта для экстремальной задачи (48) и описанию некоторых его свойств, в том числе $S$-свойства (21). Схема изложения следующая. Сначала мы докажем, что решение экстремальной задачи (48) (т. е. стационарный компакт $F$ ) можно искать среди тех допустимых компактов, которые лежат вне максимального эллипса голоморфности функции $f$. Затем, пользуясь тем, что такое подсемейство предкомпактно в хаусдорфовой топологии, мы установим существование допустимого компакта, на котором достигается величина (48). Затем с помощью вариационного метода (см. [57], а также [73]) доказывается, что этот экстремальный компакт является замыканием критических траекторий некоторого квадратичного дифференциала. Отсюда уже вытекает $S$-свойство (21). Отметим, что существует и другой подход к задаче о существовании и свойствах стационарного компакта. Этот подход основан на явном описании такого компакта в терминах некоторой алгебраической функции (см., например, [60], [74], [75]). 
Итак, докажем сначала, что существует компакт $F \in \mathscr{K}_{f}-$ решение задачи (48), принадлежащий внешности $G_{\rho}^{c}=\overline{\mathbb{C}} \backslash G_{\rho}$ максимальной канонической области голоморфности $G_{\rho}:=\{z:|\Phi(z)|<\rho\}$ функции $f$. Для этого определим "внешнее" проектирование $K^{*}$ произвольного компакта $K \in \mathscr{K}_{f}$ вдоль гипербол, соответствующих отрезку $E=[-1,1]$, следующим образом. Если точка $z$ принадлежит компакту $G_{\rho}^{c}$, то $z^{*}=z$. Пусть теперь $z \in G_{\rho} \backslash E$. Тогда через эту точку проходит единственная гипербола с фокусом в точке +1 или -1 ; так как точка $z$ не лежит на отрезке $E$, то она принадлежит верхней или нижней ветви этой гиперболы. Пусть $z^{*} \in \gamma_{\rho}-$ (единственная) точка пересечения этой ветви с максимальным эллипсом голоморфности $\gamma_{\rho}=\partial G_{\rho}$ функции $f$. Ясно, что при таком проектировании $K^{*} \in \mathscr{K}_{f}$ для любого $K \in \mathscr{K}_{f}$.

Справедлива следующая лемма.

Лемма 1. Пусть параметр $\theta$ принадлежит отрезку $[0,1]$. Тогда для любого компакта $K \in \mathscr{K}_{f}$ имеем: $K^{*} \in \mathscr{K}_{f} u I_{\varphi}\left(K^{*} ; \lambda_{K^{*}}\right) \geqslant I_{\varphi}\left(K ; \lambda_{K}\right)$.

ДокАзАтЕльство. Для смешанного ядра (см. (27)) имеем

$$
\theta \log \frac{1}{|z-t|}+g_{E}(z, t)+\theta \varphi(z)+\theta \varphi(t)=\log \left\{\frac{e^{\theta g_{E}(z, \infty)+\theta g_{E}(t, \infty)}}{|z-t|} e^{g_{E}(z, t)}\right\} .
$$

Для произвольных различных точек $z, t \in \mathbb{C} \backslash E$ положим

$$
d_{\theta}(z, t):=|z-t| e^{-\theta g_{E}(z, \infty)-\theta g_{E}(t, \infty)} e^{-g_{E}(z, t)} .
$$

Если теперь для $N$ произвольных попарно различных точек $z_{1}, \ldots, z_{N}$ ввести величину

$$
d_{\theta}\left(z_{1}, \ldots, z_{N}\right):=\prod_{1 \leqslant i<j \leqslant N} d_{\theta}\left(z_{i}, z_{j}\right)
$$

и для произвольного невырожденного компакта $K$ определить

$$
d_{\theta}(K ; N):=\max _{z_{1}, \ldots, z_{N} \in K} d_{\theta}\left(z_{1}, \ldots, z_{N}\right),
$$

то стандартным образом можно показать (см. [55; гл. II, §3]), что существует предел

$$
d_{\theta}(K):=\lim _{N \rightarrow \infty}\left(d_{\theta}(K ; N)\right)^{2 /(N(N+1))}
$$

и для этой величины $d_{\theta}(K)$ имеем

$$
d_{\theta}(K)=e^{-I_{\varphi}\left(K ; \lambda_{K}\right)} .
$$

С учетом (52) остается доказать, что при $\theta \in[0,1]$ для произвольного множества $K \subset \mathbb{C} \backslash E$ справедливо неравенство $d_{\theta}\left(K^{*}\right) \leqslant d_{\theta}(K)$.

Для $z \in \overline{\mathbb{C}} \backslash E$ положим $z=(\zeta+1 / \zeta) / 2$, где $|\zeta|<1$. Тогда введенная выше операция внешнего проектирования вдоль гипербол в плоскости $\mathbb{C}_{z}$ эквивалентна радиальному проектированию внутрь единичного круга $|\zeta|<1$ в плоскости $\mathbb{C}_{\zeta}$, а для функций Грина отрезка $E$ имеем:

$$
g_{E}(z, \infty)=\log \frac{1}{|\zeta|}, \quad g_{E}(z, t)=\log \left|\frac{1-\bar{\xi} \zeta}{\zeta-\xi}\right|
$$


где $t=(\xi+1 / \xi) / 2,|\xi|<1$. С учетом (53) для смешанного ядра (27) имеем:

$$
\begin{aligned}
& \theta \log \frac{1}{|z-t|}+g_{E}(z, t)+\theta \varphi(z)+\theta \varphi(t) \\
& \quad=\theta \log \frac{e^{g_{E}(z, \infty)} e^{g_{E}(t, \infty)}}{|z-t|}+g_{E}(z, t) \\
& \quad=\text { const }-\left\{\theta \log (|\zeta-\xi||1-\zeta \xi|)+\log \left|\frac{\zeta-\xi}{1-\zeta \bar{\xi} \mid}\right|\right\} .
\end{aligned}
$$

Так как функция $f$ вещественна на $E$ и множество $\Sigma$ симметрично относительно вещественной прямой, то можно рассматривать только такие допустимые компакты $K \in \mathscr{K}_{f}$, которые симметричны относительно $\mathbb{R}$. В силу единственности равновесной меры, мера $\lambda_{K}$ тоже симметрична. Следовательно, функционал энергии (45) не изменится, если в (53) мы уберем знак комплексного сопряжения и полученное выражение в качестве ядра подставим в (45), или наоборот, заменим в ядре выражение $|1-\zeta \xi|$ на $|1-\zeta \bar{\xi}|$. Ту же самую операцию мы можем проделать и в (54). В результате приходим к функции

$$
\rho_{\theta}\left(\zeta_{1}, \zeta_{2}\right):=\left|\zeta_{1}-\zeta_{2}\right|^{2 \theta}\left(\frac{\left|\zeta_{1}-\zeta_{2}\right|}{\left|1-\zeta_{1} \bar{\zeta}_{2}\right|}\right)^{1-\theta}, \quad \theta \in[0,1]
$$

определенной для точек из единичного круга, $\zeta_{1}, \zeta_{2} \in \mathbb{D}$. Таким образом, задача (48) о максимуме равновесной энергии в классе допустимых компактов $\mathscr{K}_{f}$ в плоскости $\mathbb{C}_{z}$ сводится (см. (52)) к задаче о минимуме обобщенного трансфинитного диаметра $d_{\theta}\left(K_{\zeta}\right)$ в соответствующем классе компактов в плоскости $\mathbb{C}_{\zeta}$. В частных случаях метрика $\rho_{\theta}\left(\zeta_{1}, \zeta_{2}\right),\left|\zeta_{1}\right|,\left|\zeta_{2}\right|<1$, имеет вид:

(1) $\rho_{0}\left(\zeta_{1}, \zeta_{2}\right)=\frac{\left|\zeta_{1}-\zeta_{2}\right|}{\left|1-\zeta_{1} \bar{\zeta}_{2}\right|}$ для $\theta=0$ - гиперболическая псевдометрика;

(2) $\rho_{1}\left(\zeta_{1}, \zeta_{2}\right)^{1 / 2}=\left|\zeta_{1}-\zeta_{2}\right|$ для $\theta=1$ - обычная евклидова метрика.

Теперь уже непосредственными вычислениями проверяется, что в случае $\theta \in[0,1]$ из-за указанного выше вида метрики (55) применим стандартный метод внутреннего радиального проектирования: при радиальном проектировании к центру единичного круга $\mathbb{D}$ (на круг меньшего радиуса $r \in(0,1)$ ) расстояние между двумя точками не увеличивается. Точнее, прямыми вычислениями проверяется следующий факт. Если точки $\zeta_{1}, \zeta_{2}$ лежат в круге $|\zeta|<r, 0<r<1$, то при $\theta \in[0,1]$ для любых $t_{1}, t_{2} \in(1,1 / r)$ имеем: $\rho_{\theta}\left(\zeta_{1}, \zeta_{2}\right) \leqslant \rho_{\theta}\left(t_{1} \zeta_{1}, t_{2} \zeta_{2}\right)$.

Непосредственно из этого факта вытекает, что так как множество $\Sigma_{\zeta}$, соответствующее множеству особых точек $\Sigma_{z}$, принадлежит единичному кругу $|\zeta|<1$, то существует экстремальный компакт $F(\theta)$ - решение задачи (48), лежащий вне эллипса $G_{\rho}$.

Лемма 1 доказана.

ЗАмечАнИЕ 6 . При $\theta=3$ соотношение $I_{\varphi}\left(K^{*} ; \lambda_{K^{*}}\right) \geqslant I_{\varphi}\left(K ; \lambda_{K}\right)$ оказывается справедливым при дополнительном условии: $K \subset G_{\rho(f)}^{c}$ и $\rho(f)>\sqrt{2}$; см. [67]. 
Из леммы 1 вытекает, что супремум в правой части (48), а следовательно, и в правой части (47) можно рассматривать среди тех допустимых компактов $K \in \mathscr{K}_{f}$, которые лежат во внешности максимального канонического эллипса голоморфности функции $f$. Такое семейство предкомпактно в хаусдорфовой топологии. В этом семействе компактов (подсемействе $\mathscr{K}_{f}$ ) мы будем искать локальное решение экстремальной задачи (47) в предположении, что соответствующий компакт $F$ - решение задачи $(38)$ - удовлетворяет условию $D(F) \ni \infty$. Точнее, зафиксируем окрестность $U(\infty)$ бесконечно удаленной точки $z=\infty$ и в дальнейшем будем считать, что супремум в (47) можно брать по подсемейству тех компактов из $\mathscr{K}_{f}$, которые не пересекаются с $U(\infty)$.

Величина $M$ конечна (см. (48)). Существует последовательность допустимых компактов $K_{n} \in \mathscr{K}_{f}$ такая, что все $K_{n} \subset \mathbb{C} \backslash G_{\rho_{0}(f)}$ и $I_{\varphi}\left(K_{n} ; \lambda_{K_{n}}\right) \rightarrow M$. Семейство $\left\{K_{n}\right\}$ предкомпактно в хаусдорфовой топологии. Значит, существуют подпоследовательность, которую мы также будем обозначать $\left\{K_{n}\right\}$, и компакт $\widetilde{K} \subset \mathbb{C} \backslash G_{\rho_{0}(f)}$ такие, что $d_{\mathrm{H}}\left(K_{n}, \widetilde{K}\right) \rightarrow 0$ при $n \rightarrow \infty$ (здесь и далее через $d_{\mathrm{H}}(\cdot, \cdot)$ обозначается расстояние между двумя компактами в хаусдорфовой метрике).

Функция $f$ принадлежит $\mathscr{A}_{E}(\overline{\mathbb{C}} \backslash \Sigma)$ и, следовательно, имеет конечное число точек ветвления (см. определение 1). Поэтому для всех достаточно больших $n \geqslant n_{0}$ все компакты $K_{n}$ содержат в точности одни и те же точки ветвления функции $f$. Значит, это справедливо и для компакта $\widetilde{K}$. Компакт $\widetilde{K}$ симметричен относительно вещественной прямой, и функция $f$ продолжается как (однозначная) голоморфная функция в ту связную компоненту $\widetilde{D}$ дополнения к $\widetilde{K}$, которая содержит отрезок $E$. Компакт $\widetilde{K}$ состоит из конечного числа континуумов, поэтому область $\widetilde{D}$ регулярна относительно решения задачи Дирихле. Следовательно, существует функция Грина $g_{\widetilde{K}}(z, \zeta)$ для области $\widetilde{D}$.

По условию выбора последовательности $\left\{K_{n}\right\}$ имеем: $I_{\varphi}\left(K_{n} ; \lambda_{K_{n}}\right) \rightarrow M$. В силу определения величины $M$ (см. (48)) справедливо неравенство

$$
I_{\varphi}\left(\widetilde{K} ; \lambda_{\widetilde{K}}\right) \leqslant M
$$

Так как для равновесных мер $\lambda_{K}$ функционал $J\left(K ; \lambda^{K}\right)($ см. (14) при $\theta=1)$ полунепрерывен сверху по $K$ и $I_{\varphi}\left(K ; \lambda_{K}\right)=2 J\left(K ; \lambda^{K}\right)-\gamma_{E}$, то из соотношения $I_{\varphi}\left(K_{n} ; \lambda_{K_{n}}\right) \rightarrow M$ вытекает, что

$$
M=\varlimsup_{n \rightarrow \infty} I_{\varphi}\left(K_{n}, \lambda_{K_{n}}\right) \leqslant I_{\varphi}\left(\widetilde{K}, \lambda_{\widetilde{K}}\right)
$$

Отсюда и из неравенства $(56)$ получаем, что $I_{\varphi}\left(\widetilde{K}, \lambda_{\widetilde{K}}\right)=M$.

Компакт $\widetilde{K}$ состоит из конечного числа континуумов, не имеет внутренних точек и не разбивает плоскость. Доказательство этого утверждения (см. [57]) проводится по схеме, предложенной в [66].

В дальнейшем полагаем $F=\widetilde{K} \in \mathscr{K}_{f}$. Вариационным методом доказывается [57], что экстремальный компакт $F$ не разбивает плоскость, состоит из конечного числа аналитических дуг, является совокупностью замыканий критических траекторий некоторого квадратичного дифференциала и обладает $S$-свойством (21). Eдинственностъ такого компакта вытекает из сходимости последовательности $F_{n}$, установленной ниже в теореме 6 . 
3.3. Приведем теперь характеризацию компакта $F=F(1)$ с помощью двулистной римановой поверхности (ср. п. 2.4).

Пусть $K \in \mathscr{K}_{f}-$ произвольный допустимый компакт. Определим риманову поверхность $\mathfrak{R}$ уравнением $W^{2}=z^{2}-1$ и положим $\mathfrak{R}=\mathfrak{R}^{(1)} \sqcup \mathfrak{R}^{(2)} \sqcup \Gamma$, где $\Gamma-$ замкнутый цикл на $\mathfrak{R}$, проходящий через точки \pm 1 и соответствующий отрезку $[-1,1]$ при каноническом проектировании, $\mathfrak{R}^{(1)}$ - первый (открытый) лист римановой поверхности, $\mathfrak{R}^{(2)}$ - второй лист. Пусть $u\left(z^{(1)}\right)=G_{K}^{\lambda}(z), z^{(1)} \in \mathfrak{R}^{(1)}$. Тогда из условий равновесия и симметрии относительно вещественной оси вытекает, что функция $u$ гармонически продолжается на второй лист $\mathfrak{R}^{(2)}$ римановой поверхности формулой $u\left(z^{(2)}\right)=w^{K}-V^{\lambda}(z)$. Полученная функция $u(\mathbf{z})$, $\mathbf{z} \in \mathfrak{R}$, обладает следующими свойствами: $u$ - гармоническая функция на $\mathfrak{R} \backslash\left(K^{(1)} \cup\left\{\infty^{(2)}\right\}\right)$, в точке $\mathbf{z}=\infty^{(2)}$ функция $u$ имеет логарифмическую особенность, $u(\mathbf{z})=0$ при $\mathbf{z} \in K^{(1)}$. Следовательно, $u(\mathbf{z})=g_{K^{(1)}}\left(\mathbf{z}, \infty^{(2)}\right)-$ функция Грина области $D=\mathfrak{R} \backslash K^{(1)}$ с особенностью в бесконечно удаленной точке $\mathbf{z}=\infty^{(2)}, w^{K}=\gamma^{(2)}-$ соответствующая постоянная Робена, $c(K)=e^{-w^{K}}-$ емкость множества $K^{(1)}$ (на $\mathfrak{R}$ относительно точки $\mathbf{z}=\infty^{(2)}$ ). Тем самым, задача (47) о максимуме постоянной равновесия $w^{K}$ в классе $K \in \mathscr{K}_{f}$ эквивалентна задаче о минимуме емкости $c(K)$ в этом классе. Соответствующий экстремальный компакт $\widetilde{F}^{(1)}$ является компактом минимальной емкости (на $\mathfrak{R}$ относительно точки $\left.\mathbf{z}=\infty^{(2)}\right)$, определяется однозначно и вполне характеризуется $S$-свойством:

$$
\frac{\partial g_{\widetilde{F}^{(1)}}\left(\zeta^{(1)}, \infty^{(2)}\right)}{\partial n_{+}}=\frac{\partial g_{\widetilde{F}^{(1)}}\left(\zeta^{(1)}, \infty^{(2)}\right)}{\partial n_{-}}, \quad \zeta^{(1)} \in \widetilde{F}_{0}^{(1)}
$$

(см. [3]-[5], [10]). В силу определения функции $u$, соотношение (57) эквивалентно $S$-свойству $(21)$. Тем самым, $F=\widetilde{F}$ и $G_{F}^{\lambda}(z)=g_{\Phi(F)}(\Phi(z), 0)$ при $z \in \overline{\mathbb{C}} \backslash E$, т. е. стационарный в смысле экстремальной задачи (48) компакт $F$ совпадает с образом компакта Шталя при отображении, задаваемом функцией ЖКуковского.

\section{4. Доказательство теоремы 6}

4.1. Перейдем теперь непосредственно к изучению сходимости диагональных нелинейных АПЧ для функции класса $\mathscr{A}_{E}(\overline{\mathbb{C}} \backslash \Sigma)$.

Приведем сначала некоторые необходимые нам свойства общих полиномов Фабера, соответствующих произвольному континууму $E \Subset \mathbb{C}$ (см. [76]).

Пусть $E$ - произвольный континуум в $\mathbb{C}$, не разбивающий плоскость и не вырождающийся в точку, $\Omega=\overline{\mathbb{C}} \backslash E$. Пусть $w=\Phi(z)-$ функция, конформно (и однолистно) отображающая область $\Omega$ на внешность единичного круга $\{w$ : $|w|>1\}$ так, что $\Phi(\infty)=\infty, \Phi^{\prime}(\infty)>0, \Phi: \Omega \rightarrow\{w:|w|>1\}, z=\Psi(w)-$ обратная функция. Для произвольного $\rho>1$ пусть $\gamma_{\rho}=\{z:|\Phi(z)|=\rho\}-$ линия уровня отображающей функции $\Phi, G_{\rho}$ - внутренность кривой $\gamma_{\rho}$. Тогда полиномы Фабера $\Phi_{n}(z)=\Phi_{n}(z ; E)$ определяются по формуле

$$
\Phi_{n}(z):=\frac{1}{2 \pi i} \int_{\gamma_{\rho}} \frac{\Phi^{n}(\zeta)}{\zeta-z} d \zeta, \quad z \in G_{\rho}, \quad \rho>1
$$


$\left(\Phi_{n}(z)\right.$ - главная часть разложения функции $\Phi^{n}(z)$ в ряд Лорана в бесконечно удаленной точке $z=\infty)$.

Из (58) вытекает, что $\Phi_{n}(z)=\Phi^{n}(z)(1+o(1)), n \rightarrow \infty$, локально равномерно в $\Omega$. Следовательно, существует предел

$$
\lim _{n \rightarrow \infty}\left|\Phi_{n}(z)\right|^{1 / n}=|\Phi(z)|, \quad z \in \Omega .
$$

Из (58) и (59) легко вытекает, что если функция $f$ - голоморфная на $E$, $f \in \mathscr{H}(E)$, то $f$ разлагается в ряд Фабера (cр. (9))

$$
f(z)=\sum_{k=0}^{\infty} c_{k} \Phi_{k}(z), \quad c_{k}=c_{k}(f)
$$

сходящийся к $f$ локально равномерно в канонической области $G_{0}(f):=G_{\rho_{0}(f)}$, где

$$
\frac{1}{\rho_{0}(f)}=\varlimsup_{k \rightarrow \infty}\left|c_{k}\right|^{1 / k}<1
$$

область $G_{0}(f)$ - максимальная каноническая область голоморфности функции $f$.

В классе функций $\mathscr{H}(E)$ стандартным образом определим (линейный) oneратор Фабера $U: \mathscr{H}(E) \rightarrow \mathscr{H}(\overline{\mathbb{D}}), \mathbb{D}:=\{w:|w|<1\}$, по формуле

$$
U(f)(w)=\sum_{k=0}^{\infty} c_{k} w^{k}
$$

где $f \in \mathscr{H}(E)$ задана разложением $f(z)=\sum_{k=0}^{\infty} c_{k} \Phi_{k}(z)$. В силу (61) функция $\tilde{f}=U(f)$ принадлежит $\mathscr{H}(\overline{\mathbb{D}})$, при этом $\rho_{0}(f)=R_{0}(\tilde{f})$, где $R_{0}$ - радиус голоморфности соответствующей функции. Тем самым, $U: \mathscr{H}(E) \rightarrow \mathscr{H}(\overline{\mathbb{D}})$. Нетрудно видеть, что отображение $U$ ограничено, биективно и справедлива следующая формула обращения:

$$
f(z)=U^{-1}(\tilde{f})(z):=\frac{1}{2 \pi i} \int_{\gamma_{\rho}} \frac{\tilde{f}(\Phi(\zeta))}{\zeta-z} d \zeta, \quad z \in G_{\rho}, \quad 1<\rho<\rho_{0}(f) .
$$

Для произвольных $n, m \in \mathbb{N}_{0}=\mathbb{N} \cup\{0\}$ положим $\mathscr{R}_{n, m}(E)=\mathscr{R}_{n, m} \cap \mathscr{H}(E)$, $\mathscr{R}_{n, m}(\overline{\mathbb{D}})=\mathscr{R}_{n, m} \cap \mathscr{H}(\overline{\mathbb{D}})$. Для произвольных фиксированных $m \in \mathbb{N}$ и $\rho>1$ обозначим через $\mathscr{M}_{m}\left(G_{\rho}\right)$ класс функций $f \in \mathscr{H}(E)$, допускающих голоморфное продолжение в каноническую область $G_{\rho}$ и имеющих там ровно ${ }^{5} m$ полюсов; аналогичным образом определяется и $\mathscr{M}_{m}\left(K_{\rho}\right)$ для $\tilde{f} \in \mathscr{H}(\overline{\mathbb{D}})$, где $K_{\rho}=\{w:|w|<\rho\}$.

Справедливо следующее утверждение (см. [30], а также [31] и [32]) о свойствах оператора Фабера.

Лемма 2. Оператор Фабера $U$ отображает:

1) ри $n \geqslant m-1$ множество $\mathscr{R}_{n, m}(E)$ линейно и взаимно однозначно на множество $\mathscr{R}_{n, m}(\overline{\mathbb{D}})$, причем точка $z_{0}$ является полюсом кратности $\mu \geqslant 1$ бункции $r(z) \in \mathscr{R}_{n, m}(E)$ тогда и только тогда, когда точка $w_{0}=\Phi\left(z_{0}\right)$ является полюсом той же кратности н функции $R(w)=U(r)(w) \in \mathscr{R}_{n, m}(\overline{\mathbb{D}})$;

\footnotetext{
${ }^{5}$ Как обычно, нули и полюсы функций считаются с учетом их кратностей.
} 
2) множество $\mathscr{M}_{m}\left(G_{\rho}\right), \rho>1$, линейно и взаимно однозначно на множество $\mathscr{M}_{m}\left(K_{\rho}\right)$, причем точка $z_{0}$ является полюсом кратности $\mu \geqslant 1$ функиии $f(z) \in \mathscr{M}_{m}\left(G_{\rho}\right)$ тогда и только тогда, когда точка $w_{0}=\Phi\left(z_{0}\right)$ является полюсом той же кратности н функиии $\tilde{f}(w)=U(f)(w)$.

Это простое утверждение впервые было сформулировано в [30] (см. также [32]). Позднее эти свойства оператора Фабера и вытекающие из них следствия (в том числе аналог теоремы Монтессу) были переоткрыты другими авторами [77], [78].

Вышеуказанные свойства оператора Фабера понадобятся нам здесь для случая, когда континуум $E$ есть отрезок $[-1,1], \Psi(w)=\operatorname{Zh}(w)=(w+1 / w) / 2-$ функция Жуковского, $w=\Phi(z)=z+\sqrt{z^{2}-1}$ и выбрана такая ветвь корня, что $\Phi(z) \sim 2 z$ при $z \rightarrow \infty$.

4.2. Из следующего соотношения (см. определение (12) нелинейных АПЧ)

$$
\left(f-F_{n}\right)(z)=\text { const } \cdot T_{2 n+1}(z)+\cdots
$$

вытекает, что

$$
\left(\tilde{f}-\widetilde{F}_{n}\right)(w)=\text { const } \cdot w^{2 n+1}+\cdots,
$$

где в силу леммы 2 функция $\widetilde{F}_{n}$ принадлежит $\mathscr{R}_{n}(\overline{\mathbb{D}})$. Следовательно, рациональная функция $\widetilde{F}_{n}(w)=U\left(F_{n}\right)(w)=[n / n]_{\tilde{f}}(w)$ - диагональная аппроксимация Паде ряда $\sum_{k=0}^{\infty} c_{k} w^{k}$ порядка $n$.

Нетрудно видеть, что оператор Фабера (в нашем случае - Фабера-Чебышёва) сохраняет характер особенностей (в частности - ветвления) при переходе от функции $f$ к функции $\tilde{f}$ и устанавливает взаимно однозначное соответствие между допустимыми компактами для функций $f$ и $\tilde{f}$ (заданных в плоскостях $\mathbb{C}_{z}$ и $\mathbb{C}_{w}$ соответственно). Следовательно, при условии, что $\tilde{f} \in \mathscr{H}(\infty)$, формулу (63) можно записать в виде

$$
f(z)=R(z)+\frac{1}{2 \pi i} \oint_{F} \frac{[\tilde{f}](\Phi(\zeta))}{\zeta-z} d \zeta, \quad z \in \Omega \backslash F,
$$

где $F \in \mathscr{K}_{f}-S$-симметричный компакт для $f, R=U^{-1}(r), r$ - сумма главных частей функции $\tilde{f}$ в $\overline{\mathbb{C}}_{w} \backslash \Phi(F),[\tilde{f}]$ - голоморфная составляющая функции $\tilde{f}$ в $\overline{\mathbb{C}}_{w} \backslash \Phi(F)$ и под $\oint_{F}$ понимается интеграл по любому контуру, охватывающему $F$ и отделяющему $F$ от точки $z$ и отрезка $E$. Аналогичное $(66)$ представление справедливо и для диагональных АПЧ $F_{n}$ функции $f$.

Из первой теоремы Шталя (см. [6]) вытекает, что $[n / n]_{\tilde{f}} \stackrel{\text { сар }}{\longrightarrow} \tilde{f}$ на компактных подмножествах области $\overline{\mathbb{C}}_{w} \backslash \widetilde{F}$, где $\widetilde{F}=\Phi(F)-$ компакт Шталя ${ }^{6}$ для функции $\tilde{f}$. При этом

$$
\left|\tilde{f}(w)-[n / n]_{\tilde{f}}(w)\right|^{1 / n} \stackrel{\text { cap }}{\longrightarrow} e^{-2 g_{\tilde{F}}(w, 0)}, \quad w \in \widetilde{K} \Subset \widetilde{D},
$$

\footnotetext{
${ }^{6}$ Более точно, компакт Шталя есть множество $S=\{z: 1 / z \in \widetilde{F}\}$ и определяется относительно бесконечно удаленной точки.
} 
$\widetilde{D}=\overline{\mathbb{C}}_{w} \backslash \widetilde{F}$, и для $\widetilde{Q}_{n}-$ знаменателей аппроксимации Паде функции $\tilde{f}$ имеем:

$$
\frac{1}{n} \mu\left(\widetilde{Q}_{n}\right) \rightarrow \lambda_{\widetilde{F}}, \quad \text { где } \lambda_{\widetilde{F}}-\text { равновесная мера для } \widetilde{F} \text {. }
$$

Из леммы 2 и соотношений $(64)-(68)$ вытекает, что $F_{n} \stackrel{\text { сар }}{\longrightarrow} f$ на компактных подмножествах области $\overline{\mathbb{C}}_{z} \backslash F$, при этом в силу (67) и равенства (см. п. 3.3 ) $G_{F}^{\lambda}(z)=g_{\widetilde{F}}(\Phi(z), 0)$, справедливого при $z \in \overline{\mathbb{C}} \backslash E$, имеем

$$
\left|f(z)-F_{n}(z)\right|^{1 / n} \stackrel{\text { cap }}{\longrightarrow} e^{-2 G_{F}^{\lambda}(z)}, \quad z \in K \Subset D,
$$

и все нули полинома $Q_{n}$ за возможным исключением $o(n)$ из них притягиваются к компакту $F$. Сходимость диагональных АПЧ функции $f$ доказана. Отметим, что из сказанного выше вытекает единственность диагональных АПЧ (по соответствующей подпоследовательности) для достаточно больших $n$.

4.3. Докажем теперь утверждения о предельном распределении нулей $Q_{n}$ и точек интерполяции.

Итак, пусть нелинейная АПЧ $F_{n}$ существует при всех $n \geqslant n_{0}$, принадлежащих некоторой бесконечной последовательности $\Lambda \subset \mathbb{N}$, и $F_{n} \in \mathscr{H}(E)$. Все дальнейшие рассуждения будут проводиться для таких $n$. Из (11) получаем следующие соотношения ортогональности:

$$
\int_{E}\left(f-F_{n}\right)(x) T_{k}(x) d \tau(x)=0, \quad k=0,1, \ldots, 2 n .
$$

Функции $f$ и $F_{n}$ вещественны на $E$. Поэтому из соотношений (70) вытекает, что разность $f-F_{n}$ обращается в нуль на $E$ по меньшей мере в $(2 n+1)$-й точке. Пусть $\omega_{2 n}(z)=z^{2 n+1}+\cdots-$ полином с нулями в этих точках. Положим $F_{n}=P_{n} / Q_{n}$, тогда имеем: функция $\left(Q_{n} f-P_{n}\right) / \omega_{2 n}$ голоморфна на $E$. Пусть $U_{m}$ - полином фиксированной степени $m$ с единичным старшим коэффициентом такой, что среди нулей $U_{m}$ содержатся все точки ветвления функции $f$ с такой кратностью, что функция $U_{m} \cdot \Delta f=U_{m} \cdot\left(f_{+}-f_{-}\right)$непрерывна на $F$; здесь и далее через $\Delta f=f_{+}-f_{-}$обозначается скачок функции $f$ на дугах, составляющих компакт $F$. Ясно, что $U_{m}$ - вещественный полином, не имеющий нулей на $E$. Функция $U_{m}\left(Q_{n} f-P_{n}\right) / \omega_{2 n}$ голоморфна в $D$. Теперь стандартным способом (см., например, [10], [79]) получаем следующие соотношения:

$$
\begin{gathered}
\left(f-F_{n}\right)(z)=\frac{\omega_{2 n}(z)}{Q_{n}(z) q(z) U_{m}(z)} \frac{1}{2 \pi i} \oint_{F} \frac{Q_{n}(t) q(t) U_{m}(t) f(t) d t}{\omega_{2 n}(t)(t-z)}, \quad z \in D \\
\oint_{F} \frac{Q_{n}(t) t^{k} U_{m}(t) f(t) d t}{\omega_{2 n}(t)}=0, \quad k=0,1, \ldots, n-1-m, \quad m=\operatorname{deg} U_{m} \\
\int_{-1}^{1} \omega_{2 n}(x) x^{j} \frac{1}{Q_{n}(x) q(x) U_{m}(x)} \\
\quad \times\left\{\oint_{F} \frac{Q_{n}(t) q(t) U_{m}(t) f(t) d t}{\omega_{2 n}(t)(t-x)}\right\} d \tau(x)=0, \quad j=\overline{0,2 n}
\end{gathered}
$$

где, как и выше, под $\oint_{F}$ понимается интеграл по любому контуру, охватывающему $F$ и отделяющему $F$ от точки $z$ и отрезка $E, q$ - произвольный полином 
степени $\leqslant n-m$. Так как функция $U_{m} \cdot \Delta f$ непрерывна на $F$, то в соотношениях (71)-(73) от интеграла $\oint_{F}$ можно перейти к интегралу $\int_{F}$, а $f(t)$ заменить на $\Delta f(t)$. Кроме того, вместо полинома $q$ в эти соотношения можно подставить полином $\widetilde{Q}_{n}$, который отличается от $Q_{n}$ сомножителем, степень которого не превосходит $m$. Таким образом, получаем

$$
\begin{aligned}
\left(f-F_{n}\right)(z) & =\frac{\omega_{2 n}(z)}{Q_{n}(z) \widetilde{Q}_{n}(z) U_{m}(z)} \frac{1}{2 \pi i} \int_{F} \frac{Q_{n}(t) \widetilde{Q}_{n}(t) U_{m}(t) \Delta f(t) d t}{\omega_{2 n}(t)(t-z)}, \quad z \in D \\
& \int_{F} \frac{Q_{n}(t) t^{k} U_{m}(t) \Delta f(t) d t}{\omega_{2 n}(t)}=0, \quad k=0,1, \ldots, n-1-m, \\
\int_{-1}^{1} \omega_{2 n}(x) x^{j} \frac{1}{Q_{n}(x) \widetilde{Q}_{n}(x) U_{m}(x)} & \\
& \times\left\{\int_{F} \frac{Q_{n}(t) \widetilde{Q}_{n}(t) U_{m}(t) \Delta f(t) d t}{\omega_{2 n}(t)(t-x)}\right\} d \tau(x)=0, \quad j=\overline{0,2 n} .
\end{aligned}
$$

В силу (69) справедливо соотношение

$$
\left|f(z)-F_{n}(z)\right|^{1 /(2 n)} \stackrel{\text { cap }}{\longrightarrow} e^{-G_{F}^{\lambda}(z)} .
$$

Так как все нули полинома $\omega_{2 n}$ лежат на отрезке $E$, то (переходя при необходимости к подпоследовательности) имеем:

$$
\left|\omega_{2 n}(z)\right|^{1 /(2 n)} \rightarrow e^{-V^{\mu \omega}(z)}
$$

локально равномерно в $\mathbb{C} \backslash E$, где $\mu_{\omega} \in M(E)$. Поскольку почти все нули полиномов $Q_{n}$ и $\widetilde{Q}_{n}$ (кроме $o(n)$ из них) притягиваются к компакту $F$, то

$$
\left|Q_{n}(z)\right|^{1 / n} \stackrel{\text { cap }}{\longrightarrow} e^{-V^{\mu} Q(z)}, \quad\left|\widetilde{Q}_{n}(z)\right|^{1 / n} \stackrel{\text { cap }}{\longrightarrow} e^{-V^{\mu} Q(z)}, \quad z \in \overline{\mathbb{C}} \backslash F,
$$

где $\mu_{Q} \in M(F)$. Следовательно, из (77)-(79) получаем:

$$
V^{\mu_{Q}}(z)-V^{\mu_{\omega}}(z)+V(z)=G_{F}^{\lambda}(z), \quad z \in \overline{\mathbb{C}} \backslash(E \cup F),
$$

где

$$
V(z):=\lim _{n \rightarrow \infty} \log \left|\int_{F} \frac{Q_{n}(t) \widetilde{Q}_{n}(t) U_{m}(t) \Delta f(t) d t}{\omega_{2 n}(t)(t-z)}\right|^{1 /(2 n)} .
$$

Функция $V$ - гармоническая вне $E \cup F$ и субгармоническая на $E$. Из соотношения равновесия (80) вытекает, что разность $V-V^{\mu_{\omega}}$ непрерывно продолжается на $E$. Потенциал $V^{\mu_{\omega}}$ - супергармоническая функция на $E$, тем самым, $V^{\mu_{\omega}}$ полунепрерывна снизу на $E$. Функция $V$ полунепрерывна сверху на $E$ и отличается от $V^{\mu_{\omega}}$ на непрерывную функцию. Следовательно, обе функции $V$ и $V^{\mu_{\omega}}$ непрерывны на $E$. Теперь уже из соотношения $(76)$, используя вещественность всех входящих в это соотношение функций, стандартным методом работы [56] получаем следующее соотношение равновесия на $E$ :

$$
-2 V^{\mu_{\omega}}(x)+V^{\mu_{Q}}(x)+V(x) \equiv \text { const, } \quad x \in E .
$$


Из соотношений (80) и (82) вытекает, что

$$
V^{\mu_{\omega}}(x)+G_{F}^{\lambda}(x) \equiv \text { const, } \quad x \in E .
$$

Следовательно, в силу условий равновесия (41) имеем:

$$
V^{\mu_{\omega}}(x) \equiv V^{\lambda}(x)+\text { const }, \quad x \in E, \quad \mu_{\omega}, \lambda \in M(E) .
$$

Отсюда получаем, что $\mu_{\omega}=\lambda$.

Наконец, воспользуемся соотношениями ортогональности (75). Из $S$-свойства компакта $F$ и равенства $\mu_{\omega}=\lambda$ вытекает, что мы находимся в условиях общей теоремы 3 работы [10; §3]. Непосредственно из этой теоремы для рассматриваемого здесь частного случая внешнего поля $\psi(z)=-V^{\lambda}(z)$ вытекает, что $\mu_{Q}=\widetilde{\lambda}$ - выметание меры $\lambda$ на $F$, a $V(z) \equiv$ const.

Из сказанного выше вытекает, что соотношения

$$
\frac{1}{2 n} \mu\left(\omega_{2 n}\right) \rightarrow \lambda, \quad \frac{1}{n} \mu\left(Q_{n}\right) \rightarrow \widetilde{\lambda}
$$

имеют место для любой подпоследовательности $\Lambda \subset \mathbb{N}$. Следовательно, $\lambda$ и $\widetilde{\lambda}-$ единственные предельные точки для последовательностей нормированных мер $\frac{1}{2 n} \mu\left(\omega_{2 n}\right)$ и $\frac{1}{n} \mu\left(Q_{n}\right)$ соответственно. Тем самым все утверждения теоремы 6 доказаны.

\section{Список литературы}

[1] Дж.Л. Уолш, Интерполяиия и аппроксимация рациональными функциями в комплексной области, ИЛ, М., 1961, 508 с.; пер. с англ.: J. L. Walsh, Interpolation and approximation by rational functions in the complex domain, 3rd ed., Amer. Math. Soc. Colloq. Publ., XX, Amer. Math. Soc., Providence, RI, 1960, x+398 pp.

[2] А.А. Гончар, "О скорости рациональной аппроксимации некоторых аналитических функций”, Матем. сб., 105(147):2 (1978), 147-163; англ. пер.: А. А. Gončar, "On the speed of rational approximation of some analytic functions", Math. USSR-Sb., 34:2 (1978), 131-145.

[3] H. Stahl, "Extremal domains associated with an analytic function. I", Complex Variables Theory Appl., 4:4 (1985), 311-324.

[4] H. Stahl, "Extremal domains associated with an analytic function. II", Complex Variables Theory Appl., 4:4 (1985), 325-338.

[5] H. Stahl, "Structure of extremal domains associated with an analytic function", Complex Variables Theory Appl., 4:4 (1985), 339-354.

[6] H. Stahl, "Orthogonal polynomials with complex valued weight function. I", Constr. Approx., 2:3 (1986), 225-240.

[7] H. Stahl, "Orthogonal polynomials with complex valued weight function. II", Constr. Approx., 2:3 (1986), 241-251.

[8] H. Stahl, "Convergence of rational interpolants", Bull. Belg. Math. Soc. Simon Stevin, 1996, suppl. issue - Numerical analysis. Papers from the Conference on Special Topics in Numerical Analysis and Applied Mathematics in honor of Jean Meinguet (Louvain-la-Neuve, 1995), 11-32.

[9] H. Stahl, "The convergence of Padé approximants to functions with branch points", J. Approx. Theory, 91:2 (1997), 139-204. 
[10] А. А. Гончар, Е. А. Рахманов, "Равновесные распределения и скорость рациональной аппроксимации аналитических функций”, Матем. сб., 134(176):3(11) (1987), 306-352; англ. пер.: А. А. Gonchar, E. A. Rakhmanov, "Equilibrium distributions and degree of rational approximation of analytic functions", Math. USSR-Sb., 62:2 (1989), 305-348.

[11] А. И. Аптекарев, В. И. Буслаев, А. Мартинес-Финкельштейн, С. П. Суетин, "Аппроксимации Паде, непрерывные дроби и ортогональные многочлены”, УМН, 66:6 (2011), 37-122.

[12] R. Jentzsch, "Untersuchungen zur Theorie der Folgen analytischer Funktionen", Acta Math., 41:1 (1916), 219-251.

[13] G. Szegö, "Über die Nullstellen von Polynomen, die in einem Kreise gleichmässig konvergieren", Sitzungsber. Berl. Math. Ges., 21 (1922), 59-64.

[14] Д. В. Христофоров, "Об асимптотических свойствах интерполяционных многочленов", Матем. заметки, 83:1 (2008), 129-138; англ. пер.: D. V. Khristoforov, "On asymptotic properties of interpolation polynomials", Math. Notes, 83:1 (2008), 116-124.

[15] Е.А. Лебедева, "Об одном обобщении теоремы Р. Йенча", Матем. заметки, 88:5 (2010), 753-758; англ. пер.: E. A. Lebedeva, "A generalization of Jentzsch's theorem", Math. Notes, 88:5 (2010), 717-722.

[16] H.-P. Blatt, R. K. Kovacheva, "Growth behavior and zero distribution of rational approximants", Constr. Approx., 34:3 (2011), 393-420.

[17] E. Remes, "Sur le calcul effectif des polynômes d'approximation de Tschebyscheff", C. R. Acad. Sci. Paris, 199 (1934), 337-340.

[18] Н. И. Ахиезер, Лекиии по теории аппроксимации, М., Наука, 1965, 407 с.; англ. пер.: N. I. Achieser, Theory of approximation, Dover Publications, Inc., New York, 1992, x+307 pp.

[19] Н.И. Ахиезер, "Чебышевское направление в теории аппроксимаций", Математика XIX века, Вып. 3, ред. А. Н. Колмогоров, А. П. Юшкевич, Наука, М., 1987, 9-79.

[20] Е.Я. Ремез, Основы численных методов чебышевского приближения, Наукова думка, Киев, 1969, 624 с.

[21] В. И. Лебедев, "О нахождении многочленов наилучшего с весом приближения", Матем. сб., 199:2 (2008), 49-70; англ. пер.: V. I. Lebedev, "Finding polynomials of best approximation with weight", Sb. Math., 199:2 (2008), 207-228.

[22] В. И. Лебедев, "О тригонометрической форме чебышевских теорем об альтернансе и фазовом итерационном методе нахождения наилучших с весом приближений", Уфимск. матем. журн., 1:4 (2009), 110-118.

[23] А. Б. Богатырев, "Эффективное решение задачи о наилучшем многочлене устойчивости", Матем. сб., 196:7 (2005), 27-50; англ. пер.: А. В. Bogatyrev, "Effective solution of the problem of the optimal stability polynomial", Sb. Math., 196:7 (2005), 959-981.

[24] А. Б. Богатырёв, “Чебышёвское представление рациональных функций”, Maтем. сб., 201:11 (2010), 19-40; англ. пер.: А. B. Bogatyrev, "Chebyshev representation for rational functions", Sb. Math., 201:11 (2010), 1579-1598.

[25] J. M. Borwein, M.P. Skerritt, An introduction to modern mathematical computing. With Maple ${ }^{\mathrm{TM}}$, Springer Undergrad. Texts Math. Technol., Springer, New York, 2011, $\mathrm{xvi}+216 \mathrm{pp}$.

[26] О.Б. Арушанян, Н. И. Волченскова, С.Ф. Залеткин, "Приближенное решение обыкновенных дифференциальных уравнений с использованием рядов Чебышева", Сиб. электрон. матем. изв., 7 (2010), 122-131. 
[27] О. Б. Арушанян, Н. И. Волченскова, С. Ф. Залеткин, "О вычислении коэффициентов рядов Чебышёва для решений обыкновенных дифференциальных уравнений", Сиб. электрон. матем. изв., 8 (2011), 273-283.

[28] J.P. Boyd, Chebyshev and Fourier spectral methods, 2nd ed., Dover Publications, Mineola, NY, 2001, xvi+668 pp.

[29] J.P. Boyd, "Chebyshev expansion on intervals with branch points with application to the root of Kepler's equation: a Chebyshev-Hermite-Padé method", J. Comput. Appl. Math., 223:2 (2009), 693-702.

[30] С. П. Суетин, "О теореме Монтессу де Болора для нелинейных аппроксимаций Паде ортогональных разложений и рядов Фабера", Докл. АН СССР, 253:6 (1980), 1322-1325; англ. пер.: S.P. Suetin, "On de Montessus de Ballore's theorem for nonlinear Pade approximants of orthogonal expansions and Faber series", Soviet Math. Dokl., 22 (1980), 274-277.

[31] K. O. Geddes, "Block structure in the Chebyshev-Padé table", SIAM J. Numer. Anal., 18:5 (1981), 844-861.

[32] С.П. Суетин, "О существовании нелинейных аппроксимаций Паде-Чебышёва для аналитических функций”, Матем. заметки, 86:2 (2009), 290-303; англ. пер.: S.P. Suetin, "On the existence of nonlinear Padé-Chebyshev approximations for analytic functions", Math. Notes, 86:2 (2009), 264-275.

[33] Л.А. Книжнерман, "Аппроксимация Паде-Фабера марковских функций на вещественно-симметричных компактах", Матем. заметки, 86:1 (2009), 81-94; англ. пер.: L. A. Knizhnerman, "Padé-Faber approximation of Markov functions on real-symmetric compact sets", Math. Notes, 86:1 (2009), 81-92.

[34] Дж. Бейкер, П. Грейвс-Моррис, Аппроксимации Паде, Мир, М., 1986, 504 с.; пер. с англ.: G. A. Baker, Jr., P. Graves-Morris, Padé approximants. Part I. Basic theory, Encyclopedia of Mathematics and its Applications, 13, Addison-Wesley, Reading, MA, 1981, xx+325 pp.; Padé approximants. Part II. Extensions and applications, Encyclopedia of Mathematics and its Applications, 14, Addison-Wesley, Reading, MA, 1981, xviii+215 pp.

[35] А. А. Гончар, Е. А. Рахманов, С. П. Суетин, "О сходимости аппроксимаций Паде ортогональных разложений", Теория чисел, алгебра, математический анализ и их приложения, Сборник статей. Посвящается 100-летию со дня рождения Ивана Матвеевича Виноградова, Тр. МИАН, 200, Наука, М., 1991, 136-146; англ. пер.: А. A. Gonchar, E. A. Rakhmanov, S.P. Suetin, "On the convergence of Padé approximation of orthogonal expansions", Proc. Steklov Inst. Math., 200 (1993), 149-159.

[36] A. A. Gonchar, E. A. Rakhmanov, S. P. Suetin, "On the rate of convergence of Padé approximants of orthogonal expansions", Progress in approximation theory (Tampa, FL, 1990), Springer Ser. Comput. Math., 19, Springer, New York, 1992, 169-190.

[37] А.А. Гончар, Е.А. Рахманов, С.П. Суетин, О сходимости аппроксимаций Чебышёва-Паде для вещественных алгебраических функиий, 2010, 10 с., arXiv: 1009.4813.

[38] А.А. Гончар, Е.А. Рахманов, С.П. Суетин, Сходимость нелинейных аппроксимаций Паде-Чебышёва для многозначных аналитических функиий, вариация равновесной энергии и $S$-свойство стачионарных компактов, 2010, 49 с., arXiv: 1012.0170 .

[39] А. А. Гончар, Е. А. Рахманов, С. П. Суетин, "О сходимости аппроксимаций ПадеЧебышёва для аналитических функций с конечным числом точек ветвления", Докл. РАН, 2012 (в печати).

[40] О.Л. Ибряева, “Достаточное условие единственности линейной аппроксимации Паде-Чебышёва", Известия Челябинского научного иентра, 2002, № 4(17), 1-5; http://csc.ac.ru/ej/issue/ru/18. 
[41] Р. Варга, Функииональный анализ и теория аппроксимачии в численном анализе, Мир, М., 1974, 126 с.; пер. с англ.: R. S. Varga, Functional analysis and approximation theory in numerical analysis, CBMS-NSF Regional Conf. Ser. Appl. Math., 3, Society for Industrial and Applied Mathematics, Philadelphia, PA, 1971, $\mathrm{v}+76 \mathrm{pp}$.

[42] R. S. Varga, "Scientific computation on some mathematical conjectures", Approximation theory, V, Proceedings of the Fifth International Symposium on Approximation Theory (College Station, TX, 1986), eds. C. K. Chui, L. L. Schumaker, J. D. Ward, Academic Press, Boston, MA, 1986, 191-209.

[43] Л. А. Книжнерман, "Выделение полюсов потенциальных полей с помощью разложения в ряды Фурье-Чебышёва", Изв. АН СССР. Сер. физика Земли, 1984, № 11, 119-123.

[44] G. L. Litvinov, "Error autocorrection in rational approximation and interval estimates. A survey of results", Cent. Eur. J. Math., 1:1 (2003), 36-60.

[45] L. N. Trefethen, M. H. Gutknecht, "The Carathéodory-Fejér method for real rational approximation", SIAM J. Numer. Anal., 20:2 (1983), 420-436.

[46] L. N. Trefethen, M. H. Gutknecht, "Padé, stable Padé, and Chebyshev-Padé approximation", Algorithms for Approximation (Shrivenham, 1985), Inst. Math. Appl. Conf. Ser. New Ser., 10, Oxford University Press, New York, 1987, 227-264.

[47] Ю.А. Лабыч, А.П. Старовойтов, "Тригонометрические аппроксимации Паде функций с регулярно убывающими коэффициентами Фурье", Матем. сб., 200:7 (2009), 107-130; англ. пер.: Yu. A. Labych, A. P. Starovoitov, "Trigonometric Padé approximants for functions with regularly decreasing Fourier coefficients", Sb. Math., 200:7 (2009), 1051-1074.

[48] Л. М. Скворцов, "Явные стабилизированные методы Рунге-Кутты”, Ж. вычисл. матем. и матем. физ., 51:7 (2011), 1236-1250; англ пер.: L. M. Skvortsov, "Explicit stabilized Runge-Kutta methods", Comput. Math. Math. Phys., 51:7 (2011), 1153-1166.

[49] С. Пашковский, Вычислительные применения многочленов и рядов Чебышёва, Наука, M., 1983, 384 с.; пер. с польск.: S. Paszkowski, Zastosowania numeryczne wielomianów i szeregów Czebyszewa, Podstawowe Algorytmy Numeryczne, Pánstwowe Wydawnictwo Naukowe, Warsaw, 1975 (Polish), 481 pp.

[50] V.I. Buslaev, "Simple counterexample to the Baker-Gammel-Wills conjecture", East J. Approx., 7:4 (2001), 515-517.

[51] В. И. Буслаев, "О гипотезе Бейкера-Гаммеля-Уиллса в теории аппроксимаций Паде", Матем. сб., 193:6 (2002), 25-38; англ. пер.: V.I. Buslaev, "On the Baker-Gammel-Wills conjecture in the theory of Padé approximants", Sb. Math., 193:6 (2002), 811-823.

[52] H. Stahl, "Diagonal Padé approximants to hyperelliptic functions", Ann. Fac. Sci. Toulouse Math. (6), 1996, Special issue, 121-193.

[53] С. П. Суетин, "О равномерной сходимости диагональных аппроксимаций Паде для гиперэллиптических функций”, Матем. сб., 191:9 (2000), 81-114; англ. пер.: S.P. Suetin, "Uniform convergence of Padé diagonal approximants for hyperelliptic functions", Sb. Math., 191:9 (2000), 1339-1373.

[54] Д.В. Христофоров, "О сходимости диагональных аппроксимаций Паде для эллиптических функций”, Матем. сб., 200:6 (2009), 143-160; англ. пер.: D. V. Khristoforov, "On uniform approximation of elliptic functions by Padé approximants", Sb. Math., 200:6 (2009), 923-941.

[55] Н. С. Ландкоф, Основы современной теории потенииала, Наука, М., 1966, 515 с.; англ. пер.: N. S. Landkof, Foundations of modern potential theory, Grundlehren Math. Wiss., 180, Springer-Verlag, Berlin-Heidelberg-New York, 1972, 424 pp. 
[56] А. А. Гончар, Е. А. Рахманов, "Равновесная мера и распределение нулей экстремальных многочленов", Матем. сб., 125(167):1(9) (1984), 117-127; англ. пер.: A. A. Gonchar, E. A. Rakhmanov, "Equilibrium measure and the distribution of zeros of extremal polynomials", Math. USSR-Sb., 53:1 (1986), 119-130.

[57] А. Мартинес-Финкельштейн, Е. А. Рахманов, С. П. Суетин, "Вариация равновесной энергии и $S$-свойство стационарных компактов”, Матем. сб., 202:12 (2011), 113-136.

[58] К. М. Ермохин, “Технология построения разрезов методом аналитического продолжения геофизических полей”, Геоинформатика, 2010, № 2, 51-60; http:// www.geosys.ru/index.php/journal/archive.html.

[59] А. А. Гончар, Е. А. Рахманов, "О задаче равновесия для векторных потенциалов", УМH, 40:4(244) (1985), 155-156; англ. пер.: А. A. Gonchar, E. A. Rakhmanov, "On the equilibrium problem for vector potentials", Russian Math. Surveys, 40:4 (1985), $183-184$.

[60] А. И. Аптекарев, “Асимптотика аппроксимаций Эрмита-Паде для пары функций с точками ветвления", Докл. РАН, 422:4 (2008), 443-445; англ. пер.: А. I. Aptekarev, "Asymptotics of Hermite-Padé approximants for two functions with branch points", Dokl. Math., 78:2 (2008), 717-719.

[61] Дж. Дженкинс, Однолистные функиии и конформные отображения, ИЛ, М., 1962, 265 с.; пер. с англ.: J. A. Jenkins, Univalent functions and conformal mapping, Ergeb. Math. Grenzgeb. Neue Folge, 18, Reihe: Moderne Funktionentheorie, Springer-Verlag, Berlin-Göttingen-Heidelberg, 1958, vi+169 pp.

[62] Г. М. Голузин, Геометрическал теория функиий комплексного переменного, 2-е изд., Наука, М., 1966, 628 с.; англ. пер.: G. M. Goluzin, Geometric theory of functions of a complex variable, Translations of Mathematical Monographs, 26, American Mathematical Society, Providence, R. I., 1969, vi+676 pp.

[63] Г. В. Кузьмина, "Модули семейств кривых и квадратичные дифференциалы”, Тр. МИАН СССР, 139, 1980, 3-241; англ. пер.: G. V. Kuz'mina, "Moduli of families of curves and quadratic differentials", Proc. Steklov Inst. Math., 139 (1982), 1-231.

[64] А. Мартинес-Финкельштейн, Е. А. Рахманов, С. П. Суетин, "Вариация равновесной меры и $S$-свойство стационарного компакта”, УMH, 66:1(397) (2011), 183-184; англ. пер.: A. Martínez-Finkelshtein, E. A. Rakhmanov, S. P. Suetin, "Variation of the equilibrium measure and the $S$-property of a stationary compact set", Russian Math. Surveys, 66:1 (2011), 176-178.

[65] Г. В. Кузьмина, "Геннадий Михайлович Голузин и геометрическая теория функций”, Алгебра и анализ, 18:3 (2006), 3-38; англ. пер.: G. V. Kuz'mina, "Gennadii Mikhailovich Goluzin and geometric function theory", St. Petersburg Math. J., 18:3 (2007), 347-372.

[66] Е. А. Перевозникова, Е. А. Рахманов, Вариачия равновесной энергии и S-свойство компактов минимальной емкости, Препринт, М., 1994.

[67] В.И. Буслаев, А. Мартинес-Финкельштейн, С. П. Суетин, Метод внутренних вариачий и существование $S$-компактов, 2012 (в печати).

[68] А.И. Аптекарев, “Точные константы рациональных аппроксимаций аналитических функций", Матем. сб., 193:1 (2002), 3-72; англ. пер.: А. I. Aptekarev, "Sharp constants for rational approximations of analytic functions", Sb. Math., 193:1 (2002), $1-72$.

[69] A. Martínez-Finkelshtein, E. A. Rakhmanov, "On asymptotic behavior of HeineStieltjes and Van Vleck polynomials", Recent trends in orthogonal polynomials and approximation theory, Contemp. Math., 507, Amer. Math. Soc., Providence, RI, 2010, 209-232. 
[70] A. Martínez-Finkelshtein, E. Rakhmanov, "Critical measures, quadratic differentials, and weak limits of zeros of Stieltjes polynomials", Comm. Math. Phys., 302:1 (2011), 53-111.

[71] А.А. Гончар, Е.А. Рахманов, "О сходимости совместных аппроксимаций Паде для систем функций марковского типа", Теория чисел, математический анализ u ux приложения, Сборник статей. Посвящается академику Ивану Матвеевичу Виноградову к его девяностолетию, Тр. МИАН СССР, 157, 1981, 31-48; англ. пер.: A. A. Gonchar, E. A. Rakhmanov, "On the convergence of simultaneous Padé approximants for systems of functions of Markov type", Proc. Steklov Inst. Math., 157 (1983), 31-50.

[72] Е. А. Рахманов, "К асимптотике многочленов Эрмита-Паде для двух марковских функций”, Матем. сб., 202:1 (2011), 133-140; англ. пер.: Е. A. Rakhmanov, "The asymptotics of Hermite-Padé polynomials for two Markov-type functions", Sb. Math., 202:1 (2011), 127-134.

[73] С. П. Суетин, "Некоторый аналог вариационных формул Адамара и Шиффера", $T M \Phi, 2012$ (в печати).

[74] А.И. Аптекарев, В.Г. Лысов, Д.Н. Туляков, "Случайные матрицы с внешним источником и асимптотика совместно ортогональных многочленов", Матем. сб., 202:2 (2011), 3-56; англ. пер.: A. I. Aptekarev, V. G. Lysov, D. N. Tulyakov, "Random matrices with external source and the asymptotic behaviour of multiple orthogonal polynomials", Sb. Math., 202:2 (2011), 155-206.

[75] A. I. Aptekarev, A. B. J. Kuijlaars, W. Van Assche, "Asymptotics of Hermite-Padé rational approximants for two analytic functions with separated pairs of branch points (case of genus 0)", Int. Math. Res. Pap. IMRP, 2008, Art. ID rpm007, 128 pp.

[76] В. И. Смирнов, Н.А. Лебедев, Конструктивная теория функиий комплексного переменного, Наука, M., 1964, 438 с.; англ. пер.: V.I. Smirnov, N. A. Lebedev, Functions of a complex variable: Constructive theory, The M.I.T. Press, Cambridge, MA, 1968, $488 \mathrm{pp}$.

[77] S. W. Ellacott, "On the Faber transform and efficient numerical rational approximation", SIAM J. Numer. Anal., 20:5 (1983), 989-1000.

[78] S. W. Ellacott, E. B. Saff, "Computing with the Faber transform", Rational approximation and interpolation (Tampa, 1983), Lecture Notes in Math., 1105, Springer, Berlin, 1984, 412-418.

[79] А.А. Гончар, Г. Лопес Лагомасино, "О теореме Маркова для многоточечных аппроксимаций Паде”, Матем. сб., 105(147):4 (1978), 512-524; англ. пер.: A. A. Gonchar, G. López Lagomasino, "On Markov's theorem for multipoint Padé approximants", Math. USSR-Sb., 34:4 (1978), 449-459.

\section{А. А. Гончар (A. A. Gonchar)}

Математический институт им. В. А. Стеклова РАН

E. А. Рахманов (E. A. Rakhmanov)

University of South Florida;

Математический институт им. В. А. Стеклова РАН

E-mail: rakhmano@shell.cas.usf.edu

\section{С. П. Суетин (S. P. Suetin)}

Математический институт им. В. А. Стеклова РАН

E-mail: suetin@mi.ras.ru
Поступила в редакцию

08.11.2011 